\title{
Radiation and Mass Transfer Effects on MHD Free Convection Flow of a Micropolar Fluid past a Stretching Surface Embedded in a Non-Darcian Porous Medium with Heat Generation
}

\author{
S. Mohammed Ibrahim, ${ }^{1}$ T. Sankar Reddy, ${ }^{2}$ and N. Bhaskar Reddy ${ }^{3}$ \\ ${ }^{1}$ Department of Mathematics, Priyadarshini College of Engineering and Technology, Nellore 524004, Andhra Pradesh, India \\ ${ }^{2}$ Deparment of Mathematics, Annamacharya College of Engineering and Technology, Cuddapa 516001, Andhra Pradesh, India \\ ${ }^{3}$ Department of Mathematics, Sri Venkateswara University, Tirupathi 517502, Andhra Pradesh, India
}

Correspondence should be addressed to S. Mohammed Ibrahim; ibrahimsvu@gmail.com

Received 15 October 2012; Accepted 6 November 2012

Academic Editors: G. L. Aranovich, T. M. Inerbaev, and M. Manciu

Copyright (c) 2013 S. Mohammed Ibrahim et al. This is an open access article distributed under the Creative Commons Attribution License, which permits unrestricted use, distribution, and reproduction in any medium, provided the original work is properly cited.

\begin{abstract}
A comprehensive study of mass transfer and thermal radiation on a steady two-dimensional laminar flow of a viscous incompressible electrically conducting micropolar fluid past a stretching surface embedded in a non-Darcian porous medium in the presence of heat generation is analyzed numerically. The governing equations of momentum, angular momentum, energy, and species equations are solved numerically using Runge-Kutta fourth order method with the shooting technique. The effects of various parameters on the velocity, microrotation, temperature and concentration field as well as skin friction coefficient, Nusselt number and Sherwood number are shown graphically and tabulated. It is observed that the micropolar fluid helps in the reduction of drag forces and also acts as a cooling agent.
\end{abstract}

\section{Introduction}

Micropolar fluids are fluid with microstructure. They belong to a class of fluids with nonsymmetric stress tensor that we will call polar fluids. Micropolar fluids may also represent fluids consisting of rigid, randomly oriented (or spherical) particles suspended in a viscous medium, where the deformation of the particle is ignored. This constitutes a substantial generalization of the Navier-Stokes model and opens a new field of potential applications. The attractiveness and power of the model of micropolar fluids are come from the fact that it is both a significant and a simple generalization of the classical Navier-Stokes model. The theory of micropolar fluids developed by Eringen [1] and has been a field of very active research for the last few decades as this class of fluids represents, mathematically, many industrial important fluids such as paints, body fluids, polymers, colloidal fluids, suspension fluids, animal blood, liquid crystal, and so forth among the various non-Newtonian fluids models. Eringen
[2] has also developed the theory of thermomicropolar fluids by the extending theory of micropolar fluids. A thorough review of the subject of the application of micropolar fluid mechanics has been given by Lukaszewicz [3] and Ariman et al. [4]. Ahmadi [5] obtained a similarity solution for micropolar boundary layer flow over a semi-infinite plate. Jena and Mathur [6] further studied the laminar free convection flow of thermomicropolar fluids past a nonisothermal vertical plate.

Boundary-layer flow and heat transfer over a continuously stretched surface have received considerable attention in recent years. This stems from various possible engineering and metallurgical applications such as a hot rolling, wire drawing, metal and plastic extrusion, continuous casting, glass fiber production, crystal growing, and paper production. The continuous surface concept was introduced by sakiadis [7, 8]. RajaGopal et al. [9] studied a boundary layer flow non-Newtonian over a stretching sheet with a 


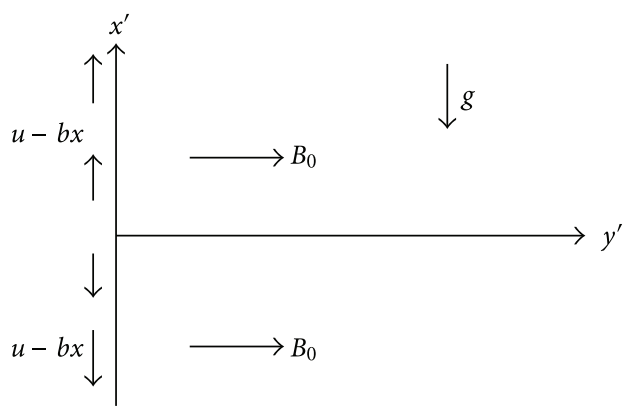

FIGURE 1: Sketch of the physical model.

uniform free stream. Hady [10] studied the solution of a heat transfer to a micropolar fluid from a nonisothermal stretching sheet with injection. $\mathrm{Na}$ and Pop [11] investigated the boundary layer flow of micropolar fluid due to a stretching wall. Hassanien et al. [12] studied a numerical solution for heat transfer in a micropolar fluid over a stretching sheet. Desseaux and Kelson [13] studied the flow of micropolar fluid bounded by stretching sheet. In all the above studies, the authors have taken the stretching sheet be an oriented in horizontal direction. However, of late the effects of MHD to the micropolar fluids problem are very important. AboEldahab and Ghonaim [14] investigated the convective heat transfer in an electrically conducting micropolar fluid at a stretching surface with uniform free stream. Pavlov [15] studied the boundary layer flow of an electrically conducting fluid due to a stretching of a plane elastic surface in the presence of a uniform transverse magnetic field. Chakrabarti and Gupta [16] extended Pavlov's work to study the heat transfer when a uniform suction is applied at the stretching surface.

Combined heat and mass transfer in fluid-saturated porous media finds applications in a variety of engineering processes such as heat exchanger devices, petroleum reservoirs, chemical catalytic reactors and processes, geothermal and geophysical engineering, moisture migration in a fibrous insulation and nuclear waste disposal, and others. Bejan and Khair [18] investigated the free convection boundary layer flow in a porous medium owing to combined heat and mass transfer. Many problems of Darcian and non-Darcian mixed convection about a vertical plate had been reported, as in Hsu and Cheng [19]; Tien and Vafai [20] investigated the free convection boundary layer flow in a porous medium owing to combined heat and mass transfer. The open literature is rich with references dealing with natural convection flow in porous medium [21-23].

In many engineering and physical problems in which fluid undergoes exothermic or endothermic reaction, it is highly important to study the effect of heat generation and absorption. Abo-Eldahb and El Aziz [17] found the heat transfer in a micropolar fluid past a stretching surface embedded in a non-Darcian porous medium with heat generation. Ishak [24] investigated the MHD flow of a micropolar fluid past a stretched permeable surface with heat generation.
Many processes in engineering areas occur at high temperatures and knowledge of radiating heat transfer becomes very important for the design of the pertinent equipment. Nuclear power plants, gas turbines, and the various propulsion devices for aircraft, missiles, satellites, and space vehicles are examples of such engineering areas. Khedr et al. [25] analyzes the radiation effects on heat transfer of a micropolar fluids through a porous medium. Abo-Eldahad and Ghonaim [26] studied the thermal boundary layer flow over a stretching sheet in a micropolar fluid with radiation. Gnaneswara [27] studied the heat generation and thermal radiation effects over a stretching sheet in a micropolar fluid. Olanrewaju et al. [28] found radiation effects on MHD flow of micropolar fluid towards a stagnation point on a vertical plate.

However the interaction of radiation with mass transfer of an electrically conducting micropolar fluid past a stretching surface has received little attention. Hence an attempt is made to investigate the radiation effects on a steady free convection flow near an isothermal vertical stretching sheet in the presence of a magnetic field, a non-Darcian porous medium and heat generation/absorption. The governing equations are transformed by using similarity transformation and the resultant dimensionless equations are solved numerically using the Runge-Kutta fourth order method with the shooting technique. The effects of various governing parameters on the velocity, temperature, concentration, skin-friction coefficient, the Nusselt number, and Sherwood number are shown in the figures and tables and analyzed in detail.

\section{Mathematical Formulation}

Let us consider a steady, two-dimensional laminar, free convection boundary layer flow of an electrically conducting and heat generating/absorbing micropolar fluid through a porous medium bounded by a vertical isothermal sheet coinciding with the plane $y=0$, where the flow confined to $y>0$. Two equal and opposite forces are introduced along the $x^{\prime}$-axis so that the sheet is linearly stretched keeping the origin fixed (see Figure 1). A uniformly distributed transverse magnetic field of strength $B_{0}$ is imposed along the $y^{\prime}$-axis. The magnetic Reynolds number of the flow is taken to be small enough so that the induced distortion of the applied magnetic field can be neglected. The viscous dissipative heat is also assumed to be negligible. It is also assumed that microscopic inertia term involving $J$ (where $J$ is the square of the characteristic length of microstructure) can be neglected for steady two-dimensional boundary layer flow in a micropolar fluid without introducing any appreciable error in the solution. Under the above assumptions and upon treating the fluid saturated porous medium as continuum [20], including the non-Darcian inertia effects, and assuming that the Boussinesq approximation is valid, the boundary layer form of the governing equations can be written as $[29,30]$

$$
\frac{\partial u^{\prime}}{\partial x^{\prime}}+\frac{\partial v^{\prime}}{\partial y^{\prime}}=0
$$




$$
\begin{aligned}
& u^{\prime} \frac{\partial u^{\prime}}{\partial x^{\prime}}+v^{\prime} \frac{\partial v^{\prime}}{\partial y^{\prime}}=v \frac{\partial^{2} u^{\prime}}{\partial y^{\prime 2}}+g \beta\left(T^{\prime}-T_{\infty}^{\prime}\right)+g \beta^{*}\left(C^{\prime}-C_{\infty}^{\prime}\right) \\
& +k_{1} \frac{\partial \sigma^{\prime}}{\partial y^{\prime}}-\frac{\sigma_{0} B_{0}^{2}}{\rho} u^{\prime}-\frac{v}{K} u^{\prime}-C u^{\prime 2} \\
& G_{1} \frac{\partial^{2} \sigma}{\partial y^{\prime 2}}-2 \sigma-\frac{\partial u^{\prime}}{\partial y^{\prime}}=0, \\
& u^{\prime} \frac{\partial T^{\prime}}{\partial x^{\prime}}+v^{\prime} \frac{\partial T^{\prime}}{\partial y^{\prime}}=\frac{k_{e}}{\rho c_{p}} \frac{\partial^{2} T^{\prime}}{\partial y^{\prime 2}}-\frac{1}{\rho c_{p}} \frac{\partial q_{r}}{\partial y^{\prime}}+\frac{Q_{0}}{\rho c_{p}}\left(T^{\prime}-T_{\infty}^{\prime}\right), \\
& u^{\prime} \frac{\partial C^{\prime}}{\partial x^{\prime}}+v^{\prime} \frac{\partial C^{\prime}}{\partial y^{\prime}}=D \frac{\partial^{2} C^{\prime}}{\partial y^{\prime 2}}
\end{aligned}
$$

subject to the boundary conditions

$$
\begin{gathered}
u^{\prime}=b x, \quad v^{\prime}=0, \quad T^{\prime}=T_{w}^{\prime}, \quad C^{\prime}=C_{w}^{\prime}, \\
\sigma=0 \quad \text { at } y=0, \\
u^{\prime} \longrightarrow u_{\infty}^{\prime}, \quad T^{\prime} \longrightarrow T_{\infty}^{\prime}, \quad C^{\prime} \longrightarrow C_{\infty}^{\prime}, \\
\sigma=0 \quad \text { as } y \longrightarrow \infty,
\end{gathered}
$$

where $x^{\prime}$ and $y^{\prime}$ are the coordinates along and normal to the sheet. $u^{\prime}$ and $v^{\prime}$ are the components of the velocity in the $x^{\prime}$ - and $y^{\prime}$-directions, respectively. $\sigma, k_{1}$, and $G_{1}$ are the microrotation component, coupling constant, and microrotation constant, respectively. $k_{e}, C, K, T^{\prime}$, and $C^{\prime}$ are the effective thermal conductivity, permeability of the porous medium, transport property related to the inertia effect, fluid temperature, and fluid concentration, respectively. $\beta, \beta^{*}$, $Q_{0}, U_{\infty}$, and $g$ are the coefficient of thermal expansion, coefficient of concentration expansion, volumetric rate of heat generation, free stream velocity, and acceleration due to gravity, respectively. $\sigma_{0}, \rho, v$, and $c_{p}$ are the electrical conductivity, density, apparent kinematic viscosity, and specific heat at constant pressure of the fluid, respectively.

By using the Rosseland approximation [31], the radiative heat flux in $y^{\prime}$ direction is given by

$$
q_{r}=-\frac{4 \sigma^{*}}{3 k^{*}} \frac{\partial T^{\prime 4}}{\partial y^{\prime}}
$$

where $\sigma^{*}$ is the Stefan-Boltzmann constant and $k^{*}$ is the mean absorption coefficient. By using (7), the energy equation (4) becomes

$$
u^{\prime} \frac{\partial T^{\prime}}{\partial x^{\prime}}+v^{\prime} \frac{\partial T^{\prime}}{\partial y^{\prime}}=\frac{k_{e}}{\rho c_{p}} \frac{\partial^{2} T^{\prime}}{\partial y^{\prime 2}}+\frac{4 \sigma^{*}}{3 k^{*} \rho c_{p}} \frac{\partial^{2} T^{\prime 4}}{\partial y^{\prime 2}} \frac{Q_{0}}{\rho c_{p}}\left(T^{\prime}-T_{\infty}^{\prime}\right) .
$$

It is convenient to make the governing equations and conditions dimensionless by using

$$
\begin{aligned}
& x=\frac{b x^{\prime}}{u_{\infty}^{\prime}}, \quad y=\frac{b y^{\prime}}{u_{\infty}^{\prime}} R, \quad u=\frac{u^{\prime}}{u_{\infty}^{\prime}}, \quad v=\frac{v^{\prime}}{u_{\infty}^{\prime}} R, \\
& R=\frac{u_{\infty}}{\sqrt{c v}}, \quad \theta=\frac{T^{\prime}-T_{\infty}^{\prime \prime}}{T_{w}^{\prime}-T_{\infty}^{\prime}}, \\
& \phi=\frac{C^{\prime}-C_{\infty}^{\prime}}{C_{w}^{\prime}-C_{\infty}^{\prime}}, \quad M=\frac{\sigma_{0} B_{0}^{2}}{\rho b}, \\
& \mathrm{Gr}=\frac{g \beta\left(T_{w}^{\prime}-T_{\infty}^{\prime}\right)}{b u_{\infty}^{\prime}}, \quad \mathrm{Gc}=\frac{g \beta^{*}\left(C_{w}^{\prime}-C_{\infty}^{\prime}\right)}{b u_{\infty}^{\prime}}, \\
& \mathrm{Q}=\frac{Q_{0}}{b \rho c_{p}}, \quad \mathrm{Sc}=\frac{v}{D}, \quad \operatorname{Pr}=\frac{\mu c_{p}}{k}, \quad F=\frac{k k^{*}}{4 \sigma^{*} T_{\infty}^{3}}, \\
& r=\frac{T_{w}^{\prime}-T_{\infty}^{\prime}}{T_{\infty}^{\prime}}, \quad \gamma=\frac{C u_{\infty}}{b}, \quad \sigma=\frac{v \sigma^{\prime}}{u_{\infty}^{2}} R, \\
& \mathrm{Da}^{-1}=\frac{v}{K b} . \quad
\end{aligned}
$$

In view of (9), the equations (1), (2), (3), (5), and (8) reduce to the following nondimensional form:

$$
\begin{gathered}
\frac{\partial u}{\partial x}+\frac{\partial v}{\partial y}=0 \\
u \frac{\partial u}{\partial x}+v \frac{\partial u}{\partial y}=\frac{\partial^{2} u}{\partial y^{2}}+N_{1} \frac{\partial \sigma}{\partial y}-M u-\frac{1}{D a} u-\gamma u^{2} \\
+\operatorname{Gr} \theta+\operatorname{Gc} \phi \\
G \frac{\partial^{2} \sigma}{\partial y^{2}}-2 \sigma-\frac{\partial u}{\partial y}=0
\end{gathered}
$$

$$
\begin{aligned}
u \frac{\partial \theta}{\partial x}+ & v \frac{\partial \theta}{\partial y} \\
= & \frac{1}{\operatorname{Pr}} \frac{\partial^{2} \theta}{\partial y^{2}}+\frac{4}{3 F \operatorname{Pr}} \\
& \times\left((1+r \theta)^{3} \frac{\partial^{2} \theta}{\partial y^{2}}+3 r(1+r \theta)^{2}\left(\frac{\partial \theta}{\partial y}\right)^{2}\right)+Q \theta
\end{aligned}
$$

$$
u \frac{\partial \phi}{\partial x}+v \frac{\partial \phi}{\partial y}=\frac{1}{\mathrm{Sc}} \frac{\partial^{2} \phi}{\partial y^{2}}
$$

The corresponding boundary conditions are

$$
\begin{aligned}
& u=x, \quad v=0, \quad \sigma=0, \quad \theta=1, \quad \phi=1 \quad \text { at } y=0, \\
& u=1, \quad \sigma=0, \quad \theta=0, \quad \phi=0 \quad \text { as } y \longrightarrow \infty,
\end{aligned}
$$


where $R$ is the Reynolds number. Proceeding with the analysis, we define a stream function $\psi(x, y)$ such that

$$
u=\frac{\partial \psi}{\partial \bar{y}}, \quad v=-\frac{\partial \psi}{\partial \bar{x}} .
$$

Now, let us consider the stream function as if

$$
\begin{aligned}
\psi(x, y) & =f(y)+x g(y) \\
\sigma & =x h(y) .
\end{aligned}
$$

In view of (16)-(17), the continuity equation (10) is identically satisfied and the momentum equation (11), angular momentum equation (12), energy equation (13), and concentration equation (14) become

$$
\begin{aligned}
\frac{\partial \psi}{\partial y} \frac{\partial^{2} \psi}{\partial x \partial y}-\frac{\partial \psi}{\partial x} \frac{\partial^{2} \psi}{\partial y^{2}} & \\
= & \frac{\partial^{3} \psi}{\partial y^{3}}+\left(M+\frac{1}{\mathrm{Da}}\right) \frac{\partial \psi}{\partial y} \\
& +\gamma\left(\frac{\partial \psi}{\partial y}\right)^{2}-\operatorname{Gr} \theta-\operatorname{Gc} \phi,
\end{aligned}
$$

$\frac{\partial \psi}{\partial y} \frac{\partial \theta}{\partial x}-\frac{\partial \psi}{\partial x} \frac{\partial \theta}{\partial y}$

$=\frac{1}{\operatorname{Pr}} \frac{\partial^{2} \theta}{\partial y^{2}}+\frac{4}{3 F \operatorname{Pr}}\left((1+r \theta)^{3} \frac{\partial^{2} \theta}{\partial y^{2}}+3 r(1+r \theta)^{2}\left(\frac{\partial \theta}{\partial y}\right)^{2}\right)$

$+Q \theta$.

$$
\begin{gathered}
G \frac{\partial^{2} \sigma}{\partial y^{2}}-2 \sigma-\frac{\partial u}{\partial y}=0 \\
\frac{\partial \psi}{\partial y} \frac{\partial \phi}{\partial x}-\frac{\partial \psi}{\partial x} \frac{\partial \phi}{\partial y}=\frac{1}{S c} \frac{\partial^{2} \phi}{\partial y^{2}}
\end{gathered}
$$

and the boundary conditions (15) become

$$
\begin{aligned}
& \frac{\partial \psi}{\partial y}=x, \quad \frac{\partial \psi}{\partial x}=0, \quad h=0, \\
& \theta=1, \quad \phi=1, \quad \text { at } y=0 \\
& \frac{\partial \psi}{\partial y} \longrightarrow 1, \quad h \longrightarrow 0, \quad \theta \longrightarrow 0, \\
& \phi \longrightarrow 0, \quad \text { as } y \longrightarrow \infty
\end{aligned}
$$

in (18) and equating coefficient of $x^{0}$ and $x^{1}$, we obtain the coupled nonlinear ordinary differential equations

$$
\begin{gathered}
f^{\prime \prime \prime}+f^{\prime \prime} g-f^{\prime} g^{\prime}-\left(M+\frac{1}{\mathrm{Da}}\right) f^{\prime}-\gamma f^{\prime 2}+\mathrm{Gr} \theta \\
+\mathrm{Gc} \phi=0, \\
g^{\prime \prime \prime}+g g^{\prime \prime}-\left(g^{\prime}\right)^{2}-\left(M+\frac{1}{\mathrm{Da}}\right) g^{\prime}-2 \gamma f^{\prime} g^{\prime} \\
+N 1 h^{\prime}=0, \\
G h^{\prime \prime}-2 h-g^{\prime \prime}=0, \\
\left(3 F+4(1+r \theta)^{3}\right) \theta^{\prime \prime}+3 \operatorname{Pr} F g \theta^{\prime} \\
+12 r(1+r \theta)^{2} \theta^{\prime 2}+3 F \operatorname{Pr} Q \theta=0, \\
\phi^{\prime \prime}+\operatorname{Sc} g \phi^{\prime}=0,
\end{gathered}
$$

where a prime denotes differentiation with respect to $y$.

In view of (17), the boundary conditions (19) reduce to

$$
\begin{aligned}
& f=0, \quad f^{\prime}=0, \quad g=0, \quad g^{\prime}=1, \quad h=1, \\
& \theta=1, \quad \phi=1, \quad \text { at } y=0 \\
& f^{\prime} \longrightarrow 1 \quad g^{\prime} \longrightarrow 0 \quad h \longrightarrow 0 \quad \theta \longrightarrow 0 \\
& \phi \longrightarrow 0, \quad \text { as } y \longrightarrow \infty .
\end{aligned}
$$

Of special significance in free convection problems are the skin-friction coefficient, the Nusselt number, and the Sherwood number.

The shear stress at the stretching surface is given by

$$
\begin{aligned}
\tau_{w} & =(\mu+k)\left(\frac{\partial u^{\prime}}{\partial y^{\prime}}\right)_{y^{\prime}=0}+k(\sigma)_{y^{\prime}=0}=\rho v b R\left(\frac{\partial u}{\partial y}\right)_{y=0} \\
& =\rho v b R\left[f^{\prime \prime}(0)+x g^{\prime \prime}(0)\right] .
\end{aligned}
$$

The skin-friction coefficient $C_{f}$ is given by

$$
C_{f}=\frac{\tau_{w}}{(1 / 2) \rho u_{\infty}^{2}}=\frac{2}{R}\left[f^{\prime \prime}(0)+x g^{\prime \prime}(0)\right] .
$$

The wall heat flux is given by

$$
\begin{aligned}
q_{w} & =-k\left(\frac{\partial T^{\prime}}{\partial y^{\prime}}\right)_{y^{\prime}=0} \\
& =-k \frac{b R}{u_{\infty}}\left(\frac{\partial T^{\prime}}{\partial y^{\prime}}\right)_{y^{\prime}=0} \\
& =-k \frac{b R}{u_{\infty}}\left(T_{w}^{\prime}-T_{\infty}^{\prime}\right) \theta^{\prime}(0) .
\end{aligned}
$$


The wall mass flux is given by

$$
\begin{aligned}
M_{w} & =-D\left(\frac{\partial C^{\prime}}{\partial y^{\prime}}\right)_{y^{\prime}=0} \\
& =-k \frac{b R}{u_{\infty}}\left(\frac{\partial C^{\prime}}{\partial y^{\prime}}\right)_{y^{\prime}=0} \\
& =-k \frac{b R}{u_{\infty}}\left(C_{w}^{\prime}-C_{\infty}^{\prime}\right) \phi^{\prime}(0) .
\end{aligned}
$$

The Nussel number

$$
\mathrm{Nu}=\frac{q_{w}}{k\left(T_{w}^{\prime}-T_{\infty}^{\prime}\right)}=\frac{b R}{u_{\infty}} \theta^{\prime}(0) .
$$

The Sherwood number

$$
\mathrm{Sh}=\frac{M_{w}}{D\left(C_{w}^{\prime}-C_{\infty}^{\prime}\right)}=\frac{b R}{u_{\infty}} \phi^{\prime}(0) .
$$

\section{Numerical Procedure}

The shooting method for linear equations is based on replacing the boundary value problem by two initial value problems and the solutions of the boundary value problem is a linear combination between the solutions of the two initial value problems. The shooting method for the nonlinear boundary value problem is similar to the linear case, except that the solution of the nonlinear problem cannot be simply expressed as a linear combination of the solutions of the two initial value problems. Instead, we need to use a sequence of suitable initial values for the derivatives such that the tolerance at the end point of the range is very small. This sequence of initial values is given by the secand method, and we use the fourth order Runge-Kutta method to solve the initial value problems.

Following Rosenhead [32] and Carnahan et al. [33], the value of $y$ at infinity is fixed at 5. The full equations (20) with the boundary conditions (21) were solved numerically using the Runge-Kutta method algorithm with a systematic guessing $f^{\prime \prime}(0), g^{\prime \prime}(0), h^{\prime}(0), \theta^{\prime}(0)$, and $\phi^{\prime}(0)$ by the shooting technique until the boundary conditions at infinity $f^{\prime}(y)$ decay exponentially to one, also $g^{\prime}(y), h(y), \theta(y)$, and $\phi(y)$ to zero. The functions $f^{\prime}, g^{\prime},-h, \theta$, and $\phi$ are shown in the figures.

\section{Results and Discussion}

As a result of the numerical calculations, the dimensionless velocity, angular velocity, temperature, and concentration distributions for the flow under consideration are obtained and their behavior has been discussed for variations in the governing parameters, namely, the thermal Grashof number Gr, solutal Grashof number Gc, magnetic field parameter $M$, radiation parameter $F$, the parameter of relative difference between the temperature of the sheet and temperature far

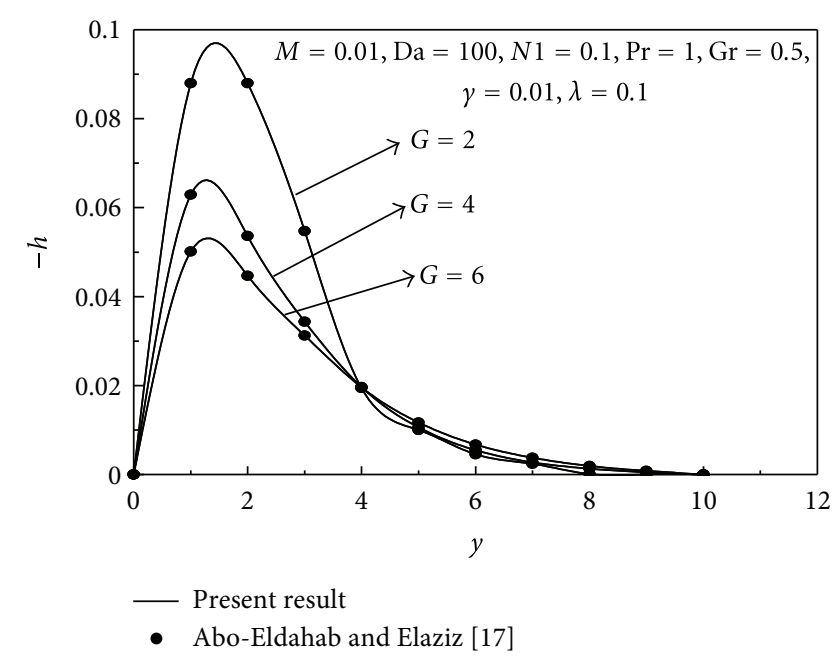

Figure 2: Comparison of angular velocity Profiles.

away from the sheet $r$, the Prandtl number Pr, heat generation parameter Q, Da Darcy number, porous medium inertia coefficient $\gamma$, vortes viscosity parameter $N 1$, microrotation parameter $G$, and the Schmidt number Sc. In the present study, the following default parametric values are adopted: $\mathrm{Gr}$ $=0.5, \mathrm{Gc}=0.5, M=0.01, \operatorname{Pr}=0.71, F=1.0, r=0.05, \mathrm{Q}=0.1$, $\gamma=0.01, N 1=0.1, G=2.0, \mathrm{Da}=100$, and $\mathrm{Sc}=0.6$. All graphs therefore correspond to these unless specifically indicated on the appropriate graph.

In order to assess the accuracy of our computed results, the present results have been compared with Abo-Eldahab and ELaziz [17] for different values of $G$ as shown in Figure 2 with $\mathrm{Gc}=0.0, F=0.0, r=0.0$, and $\mathrm{Sc}=0.0$. It is observed that the agreements with the solution of angular velocity profiles are excellent.

Figure 3(a) shows the variation of the dimensionless velocity component $f^{\prime}$ for several sets of values of thermal Grashof number Gr. As expected, it is observed that there is a rise in the velocity due to enhancement of thermal buoyancy force. The variation of the dimensionless velocity component $f^{\prime}$ for several sets of values of solutal Grashof number Gc is depicted in Figure 3(b). As expected, the fluid velocity increases and the peak value is more distinctive due to the increase in the species buoyancy force. It should be mentioned herein that the profiles of $g^{\prime},-h, \theta$ and $\phi$ were found to be insensible to change in $\mathrm{Gr}$ and $\mathrm{Gc}$, therefore, not shown herein for brevity.

The effect of variation of the magnetic parameter $M$ on the velocity ( $f^{\prime}$ and $g^{\prime}$ ), angular velocity $-h$, temperature $\theta$, and concentration $\phi$ profiles is presented in Figures 4(a)4(e) respectively. It is well known that the application of a uniform magnetic field normal to the flow direction gives rise to a force called Lorentz. This force has the tendency to slow down the velocity of the fluid and angular velocity of microrotation in the boundary layer and to increase its temperature and concentration. This is obvious from the 


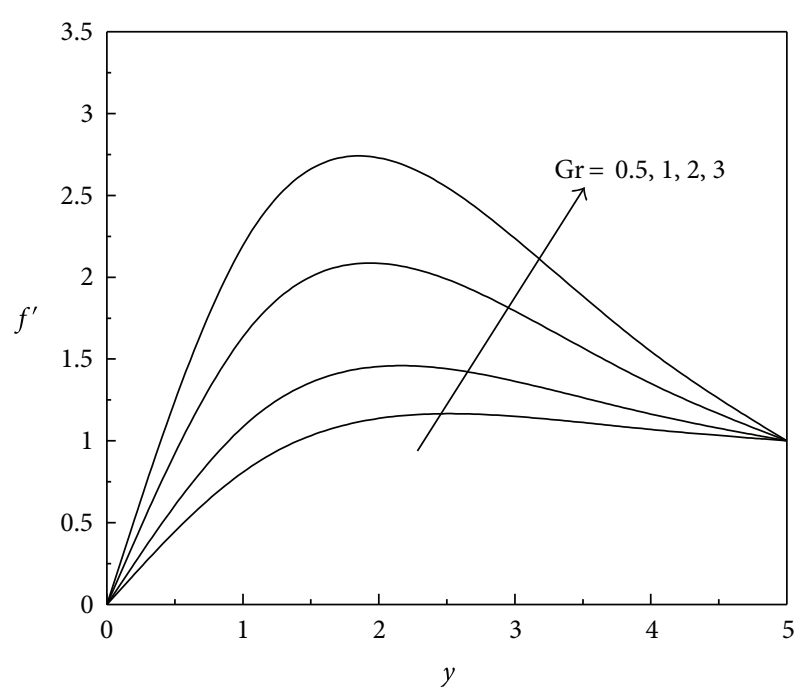

(a)

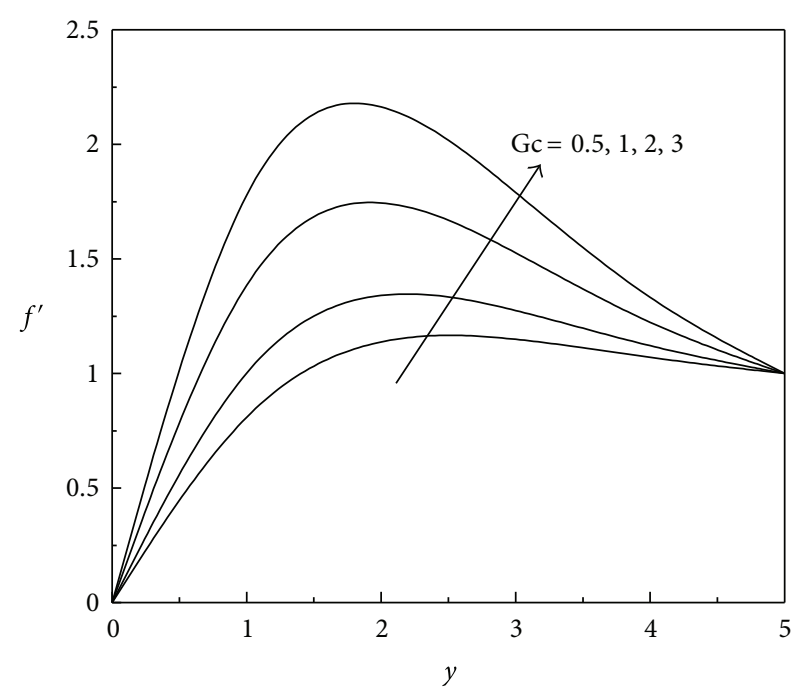

(b)

FIGURE 3: (a) Variation of the velocity component $f^{\prime}$ with Gr. (b) Variation of the velocity component $f^{\prime}$ with Gc.

TABle 1: Comparison of the present results with the literature results given by Abo-Eldahab and El Aziz [17], Gc $=0.0, F=0.0, r=0.0$, $\mathrm{Sc}=0.0 . \mathrm{Pr}=1.0, \mathrm{Da}=100, \gamma=0.01, N 1=0.1, G=2, \mathrm{Gr}=0.5$, and $\mathrm{Q}=0.1$.

\begin{tabular}{lcccr}
\hline$M$ & & $f^{\prime \prime}(0)$ & & $g^{\prime \prime}(0)$ \\
& Present & Abo-Eldahab [17] & Present & Abo-Eldahab [17] \\
\hline 0.1 & 0.67059875 & 0.6708520 & -1.0002147 & -0.998951 \\
0.2 & 0.57169871 & 0.5717493 & -1.0002075 & -0.998905 \\
\hline
\end{tabular}

decreases in the velocity profiles, angular velocity of microrotation profiles, while temperature profiles and concentration profiles increase, presented in Figures 4(a)-4(e) respectively.

Figures 5(a)-5(e) present typical profiles for the variables of the fluid's $x$-component of velocity ( $f^{\prime}$ and $g$ ), angular velocity $-h$, temperature $\theta$, and concentration $\phi$ for different values of Darcy number Da. It is noted that values of $\mathrm{Da}$ increase the fluid velocities and angular velocity increases, while temperature and concentration of the fluid decrease.

Figures 6(a)-6(e) present the typical profiles for the variables of the fluid's $\mathrm{x}$-component of velocity $\left(f^{\prime}\right.$ and $g$ ), angular velocity $-h$, temperature $\theta$ and concentration $\phi$ for different values of the porous medium inertia coefficient $\gamma$. Obviously, the porous medium inertia effects constitute resistance to the flow. Thus as the inertia coefficient increases, the resistance to the flow increases, causing the fluid flow in the porous medium to slow down and the temperature and concentration increase and, therefore, as $\gamma$ increases $f^{\prime}, g^{\prime}$, and $-h$ decreases while the temperature $\theta$ and concentration $\phi$ increase.

Figures $7(\mathrm{a})-7(\mathrm{e})$ present the typical profiles for the variables of the fluid's $x$-component of velocity ( $f^{\prime}$ and $g$ '), angular velocity $-h$, temperature $\theta$, and concentration $\phi$ for different values of the vortex viscosity parameter $N 1$. Increases in the values of $N 1$ have a tendency to increase $f^{\prime},-h, \theta$, and $\phi$ and to decrease $g$.
Figure 8 is a plot of the dimensionless angular velocity $-\mathrm{h}$ profiles for different values of the presence of the microrotation parameter $G$. The curves illustrate that, as the values of $G$ increases, the angular velocity $-h$, as expected, decreases with an increase in the boundary layer thickness as the maximum moves away from the sheet. Of course, when the viscosity of the fluid decreases the angular velocity of additive increases.

Figures 9(a) and 9(b) illustrate the respective changes in the profiles of $f^{\prime}$ and $\theta$ as the heat generation/absorption coefficient $Q$ is changed. It is clear from Figures 9(a) and 9(b) that increasing in the values of $Q$ produces increases in the velocity $f^{\prime}$ and temperature $\theta$ distributions of the fluid. This is expected since heat generation $(Q>0)$ causes the thermal boundary layer to become thicker and the fluid to be warmer. this enhances the effects of thermal buoyancy of the driving body force due to mass density variations which are coupled to the temperature distribution and therefore increasing the fluid velocity distribution. No figures for $g^{\prime},-h$ and $\phi$ are presented for the same reason as mentioned before.

Figure 10(a) Illustrates the dimensionless velocity component $f^{\prime}$ for different values of the Prandtl number Pr. The numerical results show that the effect of increasing values of the Prandtl number results in a decreasing velocity. From Figure 10(b), it is observed that an increase in the Prandtl number results in a decrease of the thermal boundary layer thickness and in general lower average temperature within 


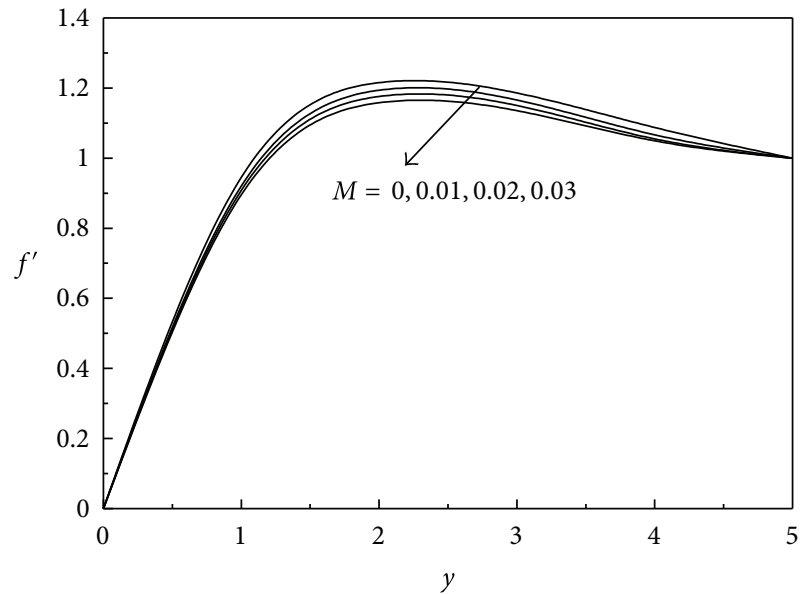

(a)

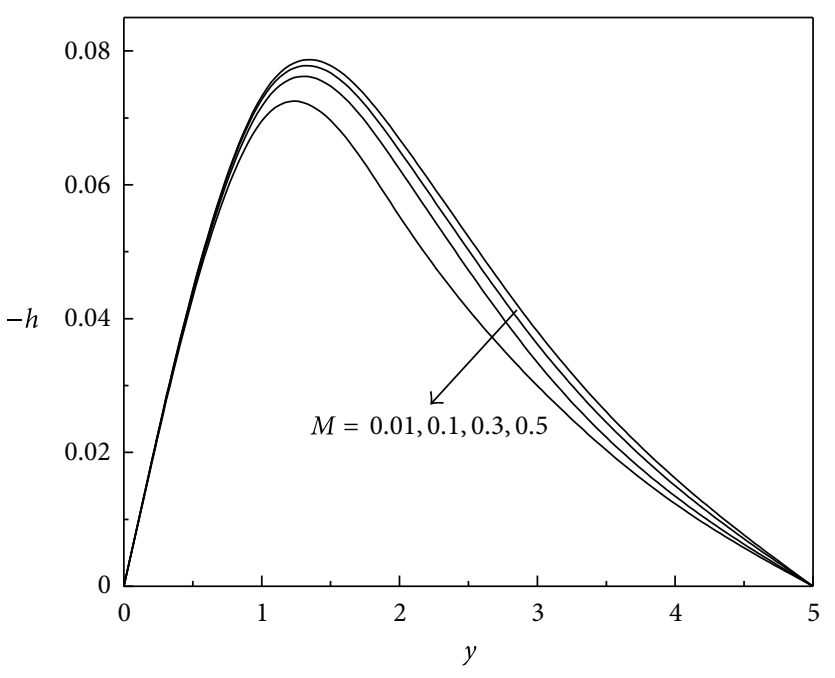

(c)

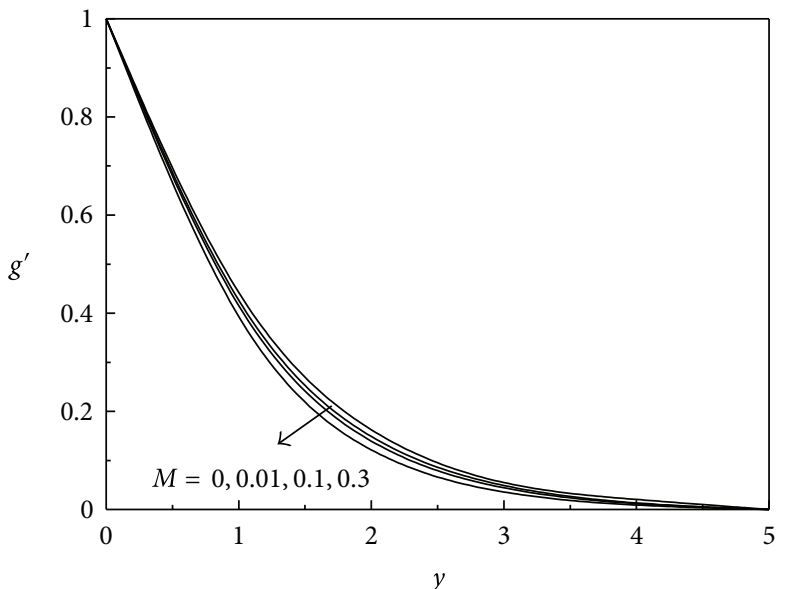

(b)

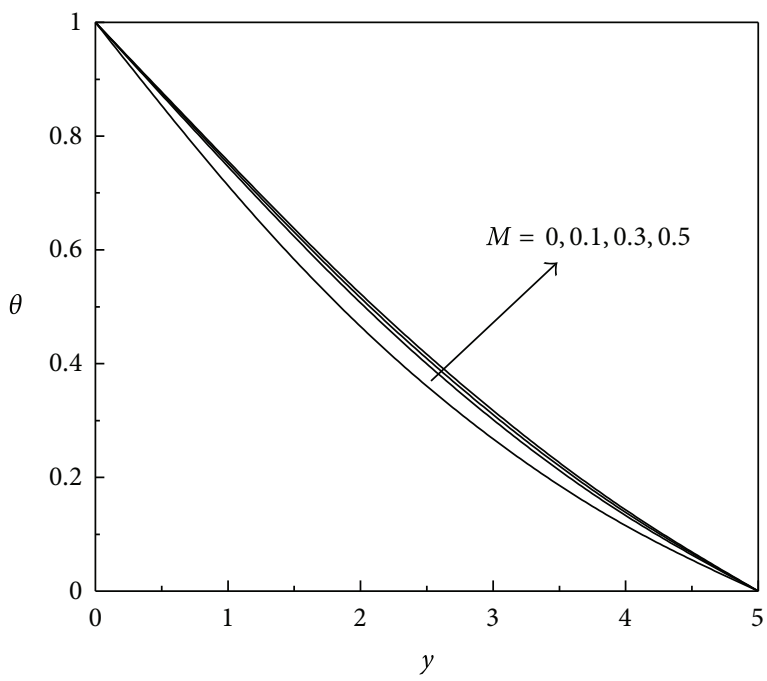

(d)

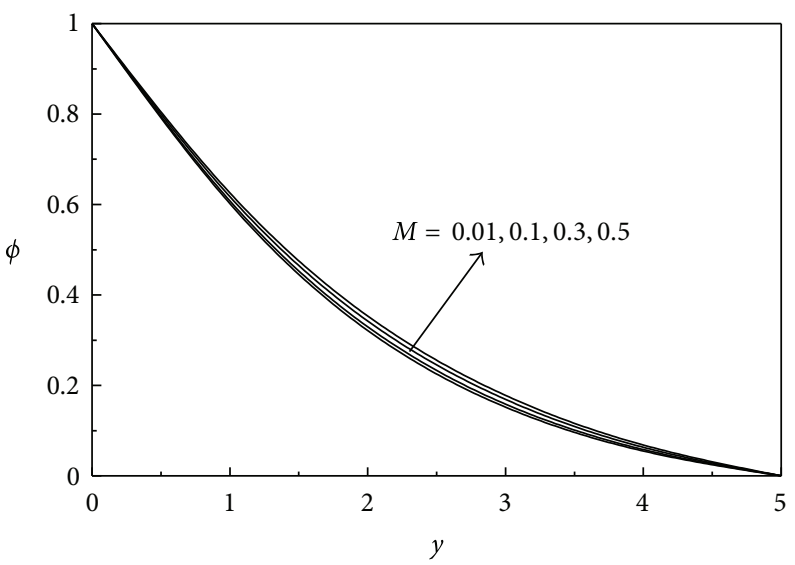

(e)

FIGURE 4: (a) Variation of the velocity component $f^{\prime}$ with $M$. (b) Variation of the velocity component $g^{\prime}$ with $M$. (c) Variation of the velocity component $-h$ with $M$. (d) Variation of the velocity component $\theta$ with $M$. (e) Variation of the velocity component $\phi$ with $M$. 


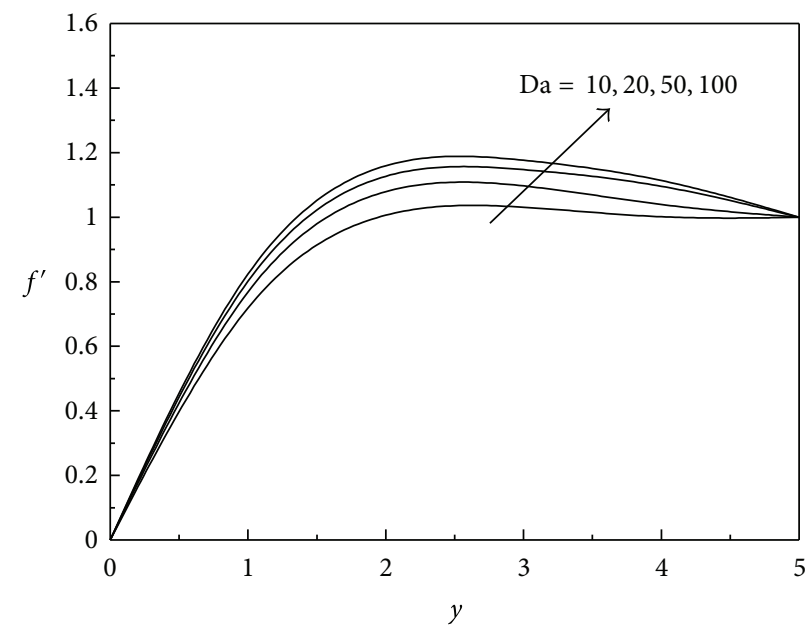

(a)

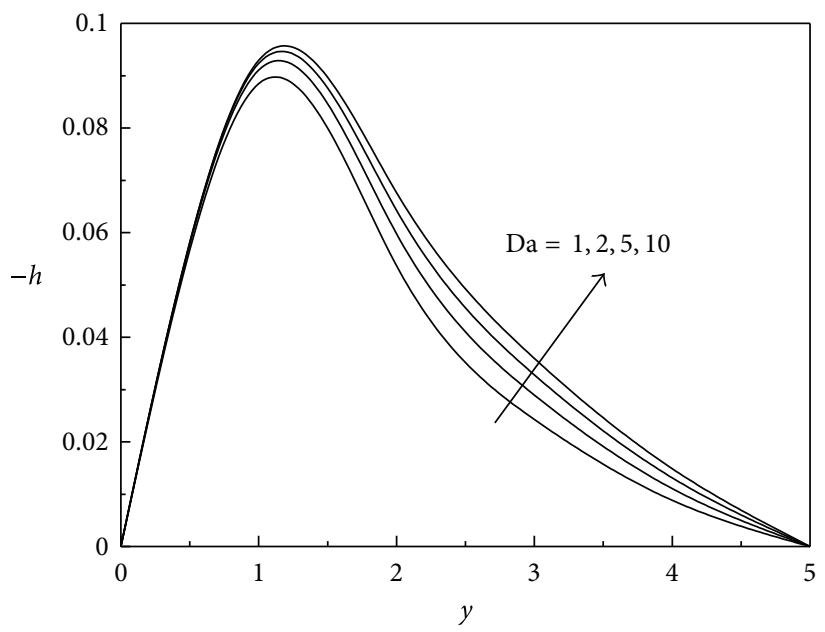

(c)

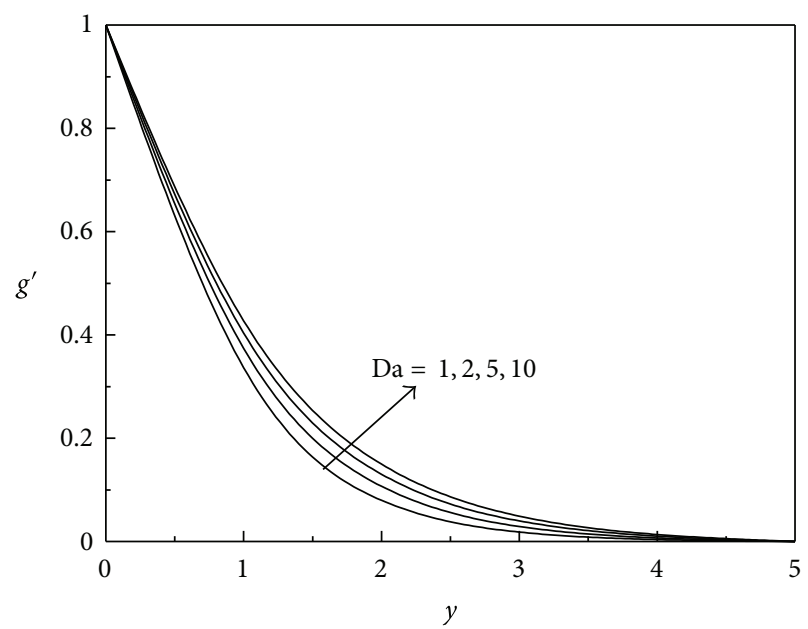

(b)

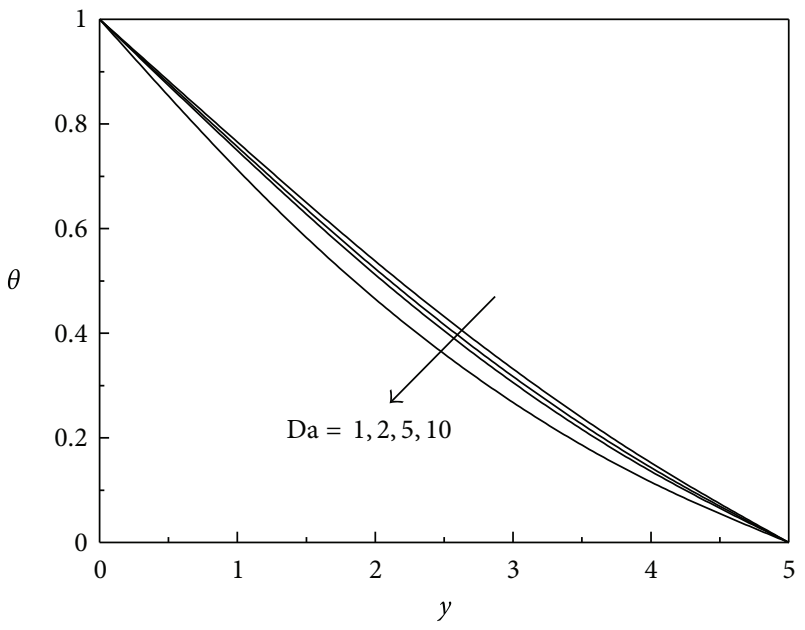

(d)

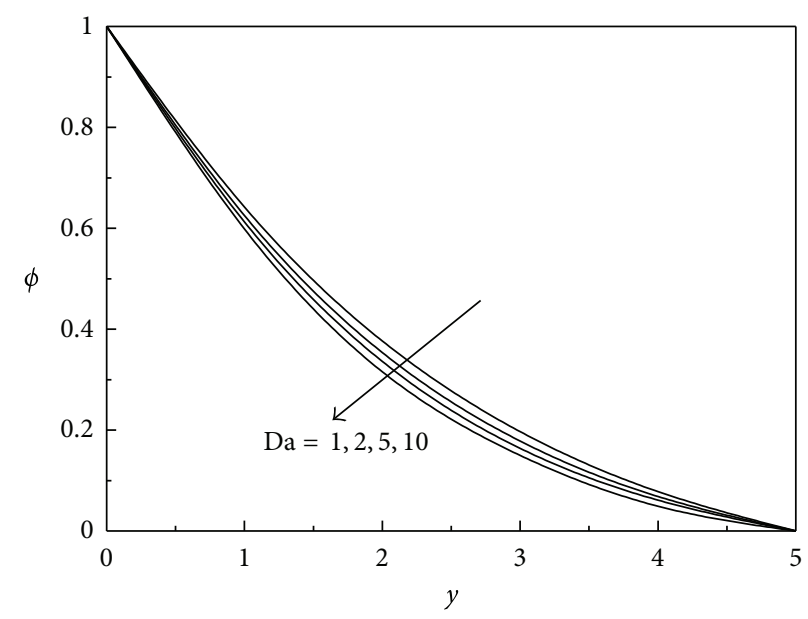

(e)

FIGURE 5: (a) Variation of the velocity component $f^{\prime}$ with Da. (b) Variation of the velocity component $g$ ' with Da. (c) Variation of the velocity component $-h$ with Da. (d) Variation of the velocity component $\theta$ with Da. (e) Variation of the velocity component $\phi$ with Da. 


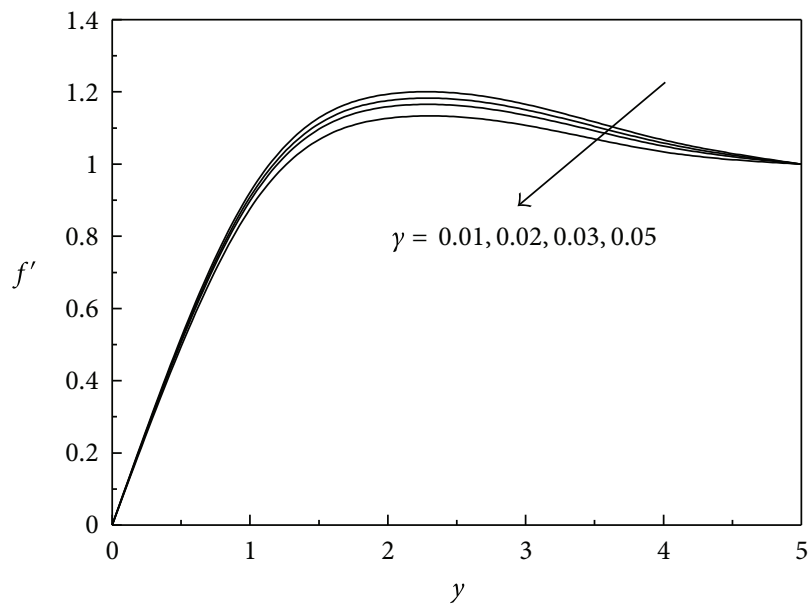

(a)

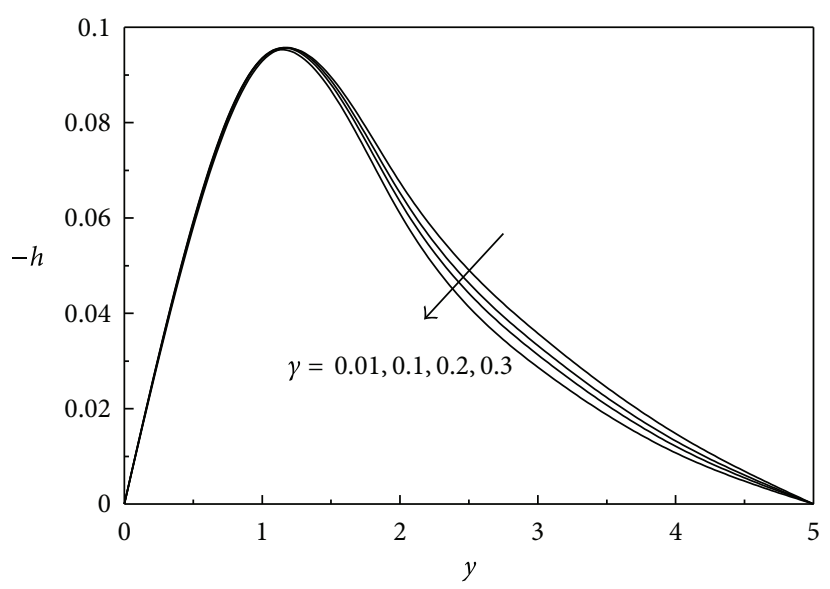

(c)

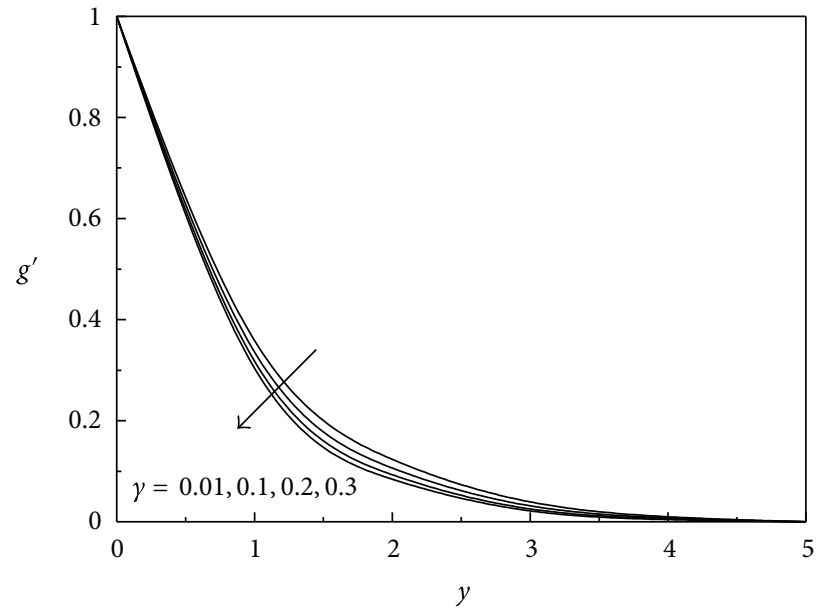

(b)

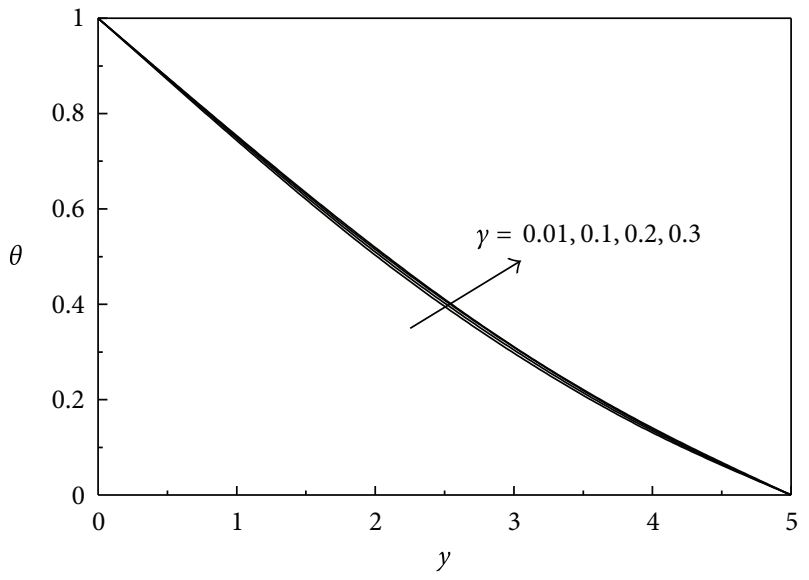

(d)

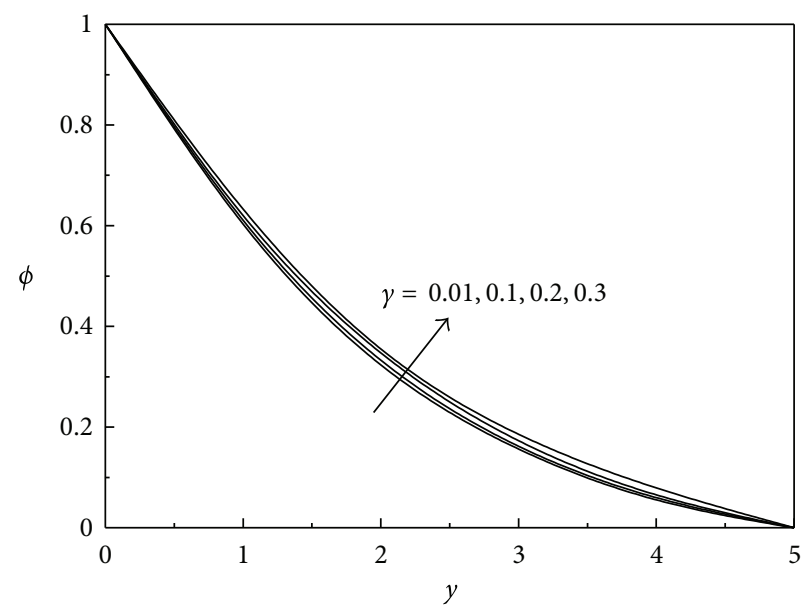

(e)

Figure 6: (a) Variation of the velocity component $f^{\prime}$ with $\gamma$. (b) Variation of the velocity component $g^{\prime}$ with $\gamma$. (c) Variation of the velocity component $-h$ with $\gamma$. (d) Variation of the velocity component $\theta$ with $\gamma$. (e) Variation of the velocity component $\phi$ with $\gamma$.

the boundary layer. The reason is that smaller values of $\operatorname{Pr}$ are equivalent to increasing the thermal conductivities, and therefore heat is able to diffuse away from the heated plate more rapidly than for higher values of Pr. Hence in the case of smaller Prandtl numbers as the boundary layer is thicker, the rate of heat transfer is reduced.

The effect of the radiation parameter $F$ on the dimensionless velocity component $f^{\prime}$ and dimensionless temperature is 


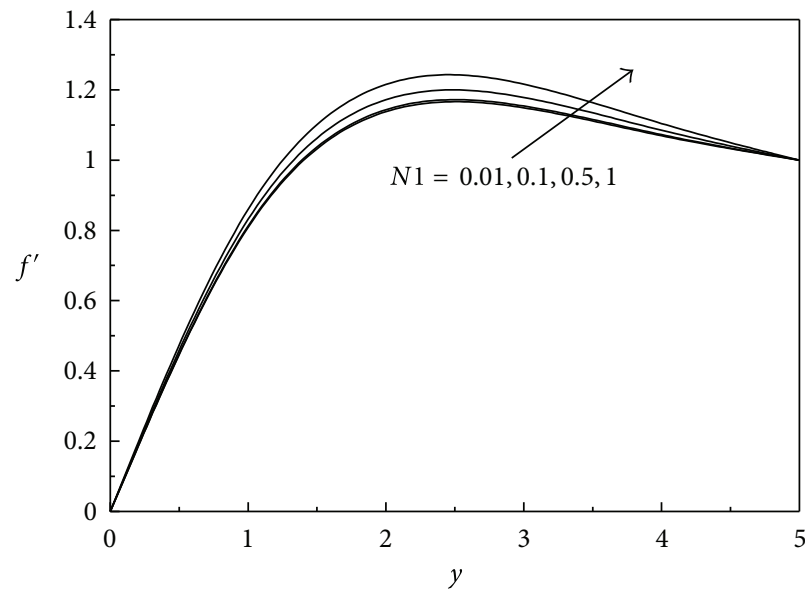

(a)

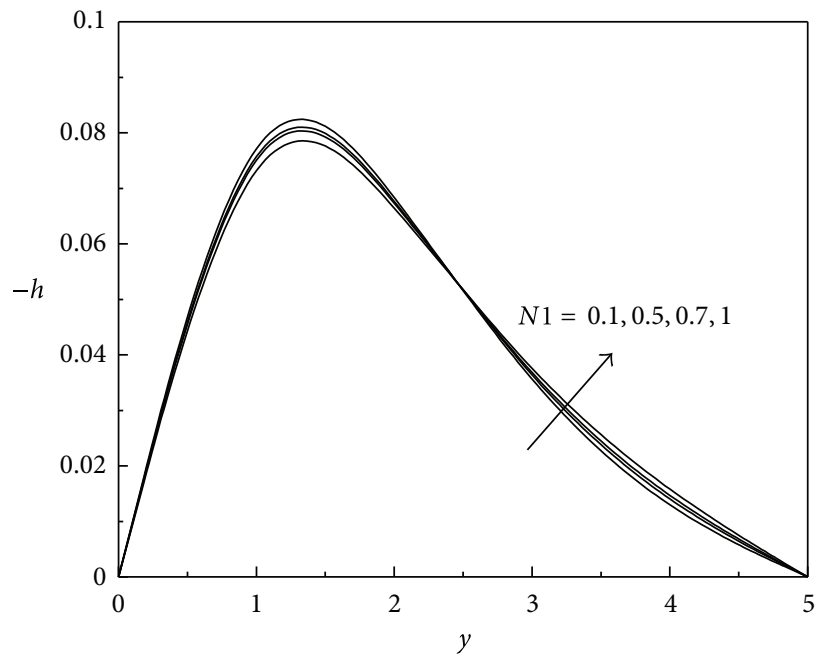

(c)

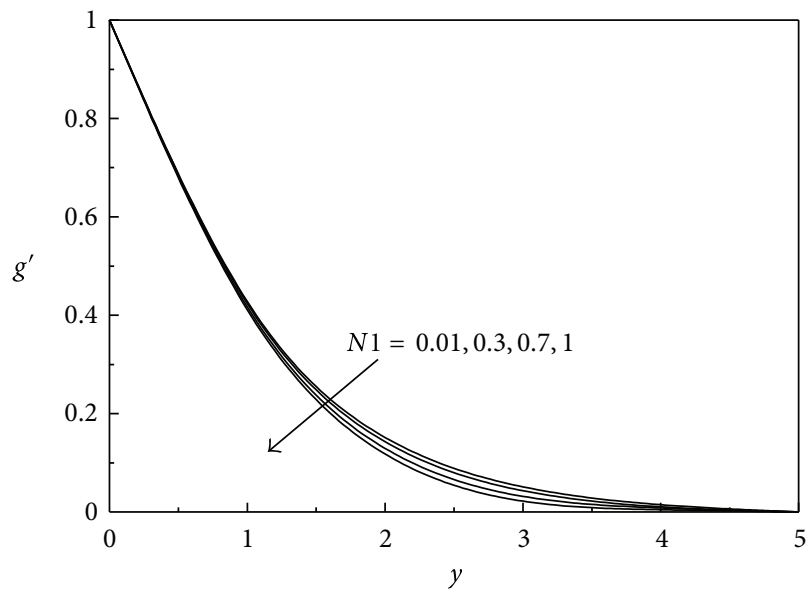

(b)

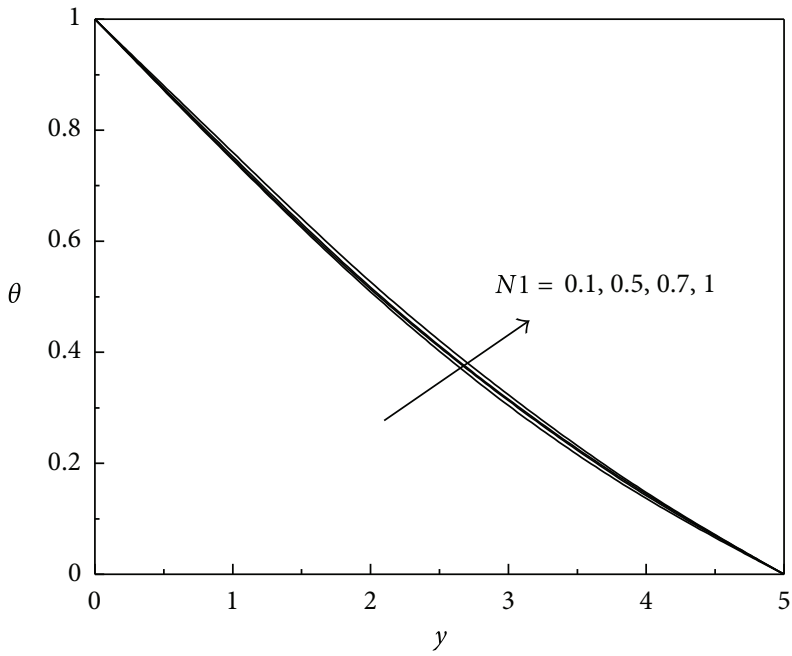

(d)

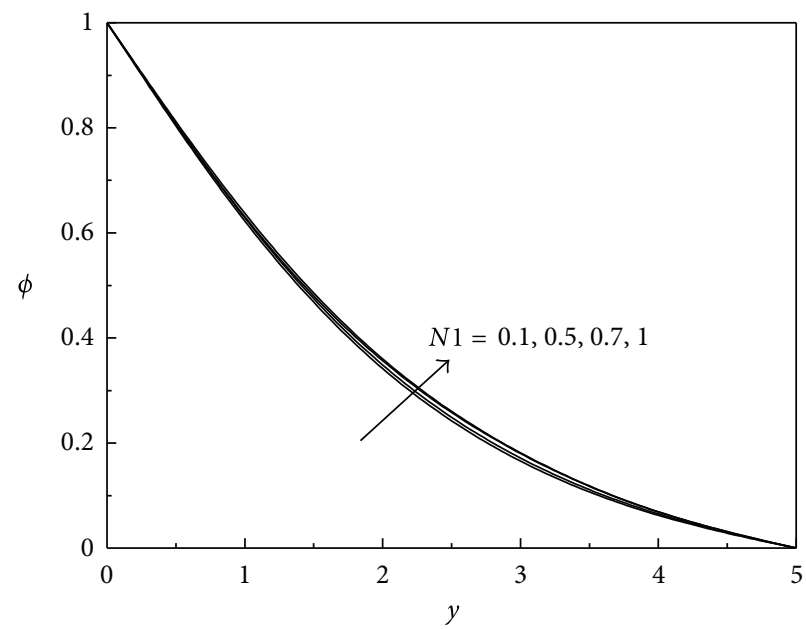

(e)

FIGURE 7: (a) Variation of the velocity component $f^{\prime}$ with $N 1$. (b) Variation of the velocity component $g$ with $N 1$. (c) Variation of the velocity component $-h$ with $N 1$. (d) Variation of the velocity component $\theta$ with $N 1$. (e) Variation of the velocity component $\phi$ with $N 1$. 
TABle 2: Variation of $f^{\prime \prime}, g^{\prime \prime},-h^{\prime}, \theta^{\prime}$, and $\phi^{\prime}$ at the plate with Gr, Gc, $M$, Da, and $\gamma$ for $\operatorname{Pr}=0.71, F=1.0, r=0.05, Q=0.1$, and Sc $=0.6$.

\begin{tabular}{cccccccccc}
\hline $\mathrm{Gr}$ & $\mathrm{Gc}$ & $M$ & $\mathrm{Da}$ & $\gamma$ & $f^{\prime \prime}(0)$ & $g^{\prime \prime}(0)$ & $h^{\prime}(0)$ & $-\theta^{\prime}(0)$ & $-\phi^{\prime}(0)$ \\
\hline 0.5 & 0.5 & 0.1 & 100 & 0.01 & 1.21865 & -1.05339 & 0.25808 & 0.27721 & 0.42336 \\
1.0 & 0.5 & 0.1 & 100 & 0.01 & 1.72256 & -1.05339 & 0.25833 & 0.276989 & 0.42336 \\
0.5 & 1.0 & 0.1 & 100 & 0.01 & 1.64847 & -1.05339 & 0.25827 & 0.277042 & 0.42336 \\
0.5 & 0.5 & 0.2 & 100 & 0.01 & 1.11425 & -1.05275 & 0.26313 & 0.272843 & 0.41783 \\
0.5 & 0.5 & 0.1 & 10 & 0.01 & 1.12368 & -1.09429 & 0.26264 & 0.273265 & 0.41836 \\
0.5 & 0.5 & 0.1 & 20 & 0.01 & 1.17407 & -1.09973 & 0.26014 & 0.275425 & 0.42110 \\
0.5 & 0.5 & 0.1 & 100 & 0.1 & 1.14886 & -1.08451 & 0.26392 & 0.271914 & 0.41678 \\
\hline
\end{tabular}

TABle 3: Variation of $f^{\prime \prime}, g^{\prime \prime},-h^{\prime}, \theta^{\prime}$, and $\phi^{\prime}$ at the plate with $G$, Pr, N1, F, Q, and Sc for Gr $=0.5$, Gc $=0.5, M=0.1$, and Da $=100$.

\begin{tabular}{ccccccccccc}
\hline$G$ & $\operatorname{Pr}$ & $N 1$ & $F$ & $\lambda$ & Sc & $f^{\prime \prime}(0)$ & $g^{\prime \prime}(0)$ & $-h^{\prime}(0)$ & $\theta^{\prime}(0)$ & $\phi^{\prime}(0)$ \\
\hline 2 & 0.71 & 0.1 & 1.0 & 0.1 & 0.6 & 1.12865 & -1.05339 & 0.25808 & 0.27721 & 0.42336 \\
4 & 0.71 & 0.1 & 1.0 & 0.1 & 0.6 & 1.21747 & -1.05339 & 0.15006 & 0.27721 & 0.42336 \\
2 & 1.0 & 0.1 & 1.0 & 0.1 & 0.6 & 1.1752 & -1.05339 & 0.25805 & 0.33548 & 0.42336 \\
2 & 0.71 & 0.4 & 1.0 & 0.1 & 0.6 & 1.22994 & -1.03823 & 0.25937 & 0.27557 & 0.42164 \\
2 & 0.71 & 0.1 & 2.0 & 0.1 & 0.6 & 1.18077 & -1.05339 & 0.25805 & 0.32959 & 0.42336 \\
2 & 0.71 & 0.1 & 1.0 & 0.3 & 0.6 & 1.28848 & -1.05366 & 0.25814 & 0.13497 & 0.42336 \\
2 & 0.71 & 0.1 & 1.0 & 0.1 & 0.78 & 1.18289 & -1.05339 & 0.25806 & 0.27723 & 0.49329 \\
\hline
\end{tabular}

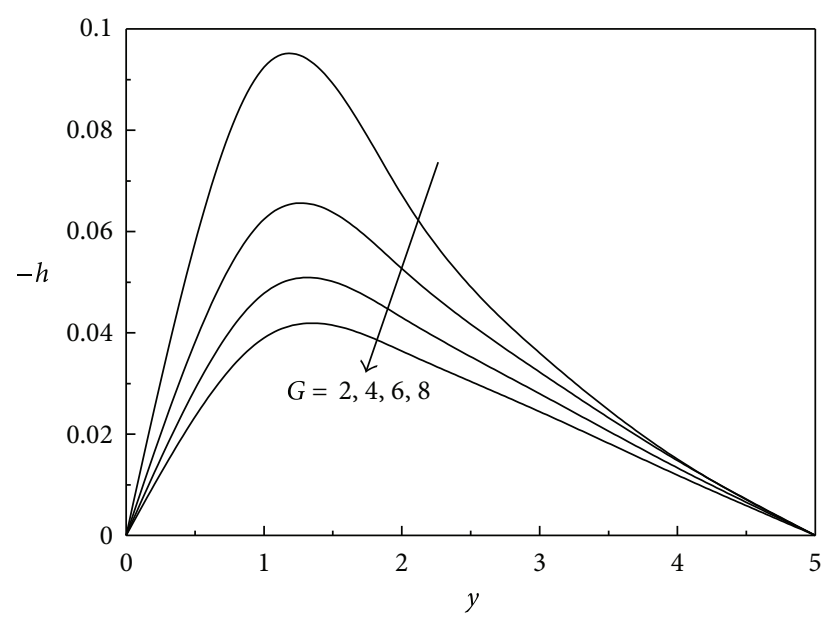

FIGURE 8: Variation of the velocity component $-h$ with $G$.

shown in Figures 11(a) and 11(b) respectively. Figure 11(a) shows that velocity component $f^{\prime}$ decreases with an increase in the radiation parameter F. From Figure 11(b) it is seen that the temperature decreases as the radiation parameter $F$ increases. This result qualitatively agrees with expectations, since the effect of radiation is to decrease the rate of energy transport to the fluid, thereby decreasing the temperature of the fluid.

The influence of the parameter of relative difference between the temperature of the sheet and the temperature far away from the sheet $r$ on dimensionless velocity $f^{\prime}$ and temperature profiles are plotted in Figures 12(a) and 12(b), respectively. Figure 12(a) shows that dimensionless velocity $f^{\prime}$ increases with an increase in $r$. It is observed that the temperature increases with an increase in $\mathrm{r}$ (Figure 12(b)).

The influence of the Schmidt number Sc on the dimensionless velocity $f^{\prime}$ and concentration profiles are plotted in Figures 13(a) and 13(b), respectively. As the Schmidt number increases the concentration decreases. This causes the concentration buoyancy effects to decrease yielding a reduction in the fluid velocity. The reductions in the velocity and concentration profiles are accompanied by simultaneous reductions in the velocity and concentration boundary layers. These behaviors are clear from Figures 13(a) and 13(b).

The accuracy of the aforementioned numerical method was also validated by direct comparisons with the numerical results reported by Abo-Eldahab and El Aziz [17]. Table 1 presents comparisons for the velocities of $f^{\prime \prime}(0)$ and $g$ (") values for various $M$ values. These comparisons show excellent agreement between the results.

Table 2 illustrates the missing wall functions for velocity, angular velocity, temperature, and concentration functions. These quantities are useful in the evaluation of wall shear stresses, gradient of angular velocity, surface heat transfer rate, and mass transfer rate. The results are obtained for $r=$ 0.05 and different values of the thermal Grashof number Gr, solutal Grashof number Gc, magnetic field parameter $M$, radiation parameter $F$, the parameter of relative difference between the temperature of the sheet and temperature far away from the sheet $r$, Prandtl number Pr, heat generation parameter $\lambda$, Darcy number $\mathrm{Da}$, porous medium inertia coefficient $\gamma$, vortes viscosity parameter $N 1$, microrotation parameter $G$, and Schmidt number Sc. Table 1 indicates that increasing the values of the Grashof number Gr and solutal Grashof number Gc results in an increase in the values of $f^{\prime \prime}(0)$. This is because as $\mathrm{Gr}$ and Gc increase, the 


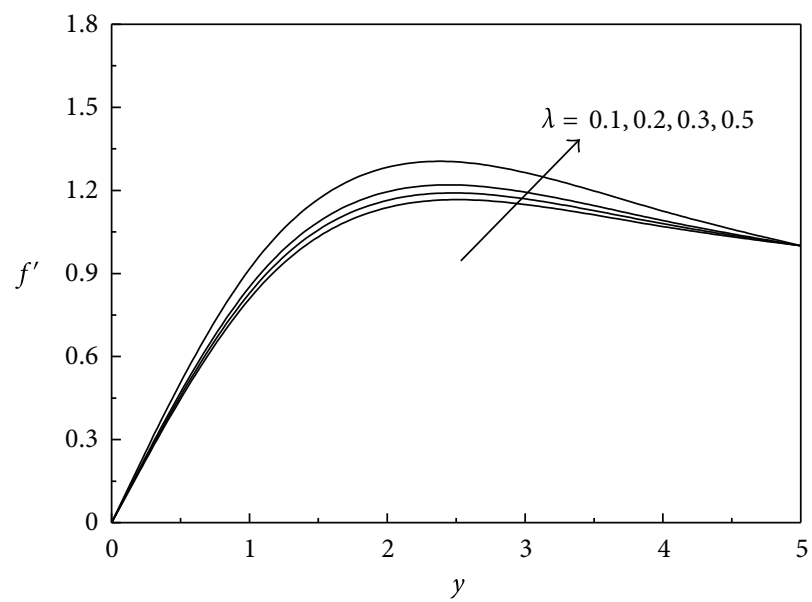

(a)

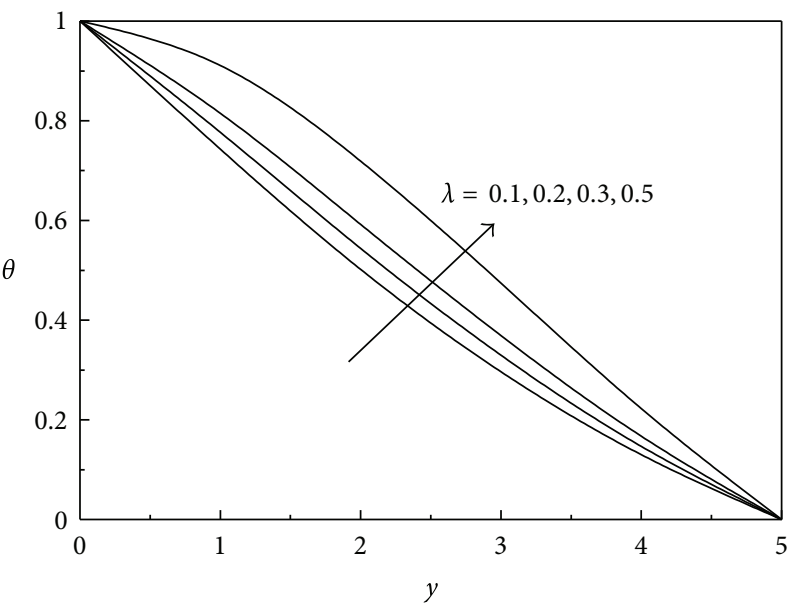

(b)

FIGURE 9: (a) Variation of the velocity component $f^{\prime}$ with Q. (b) Variation of the velocity component $\theta$ with Q.

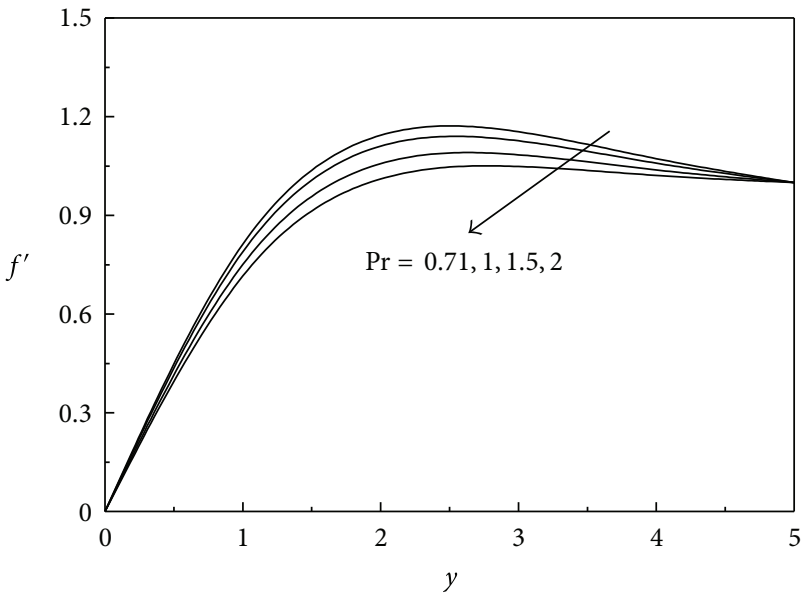

(a)

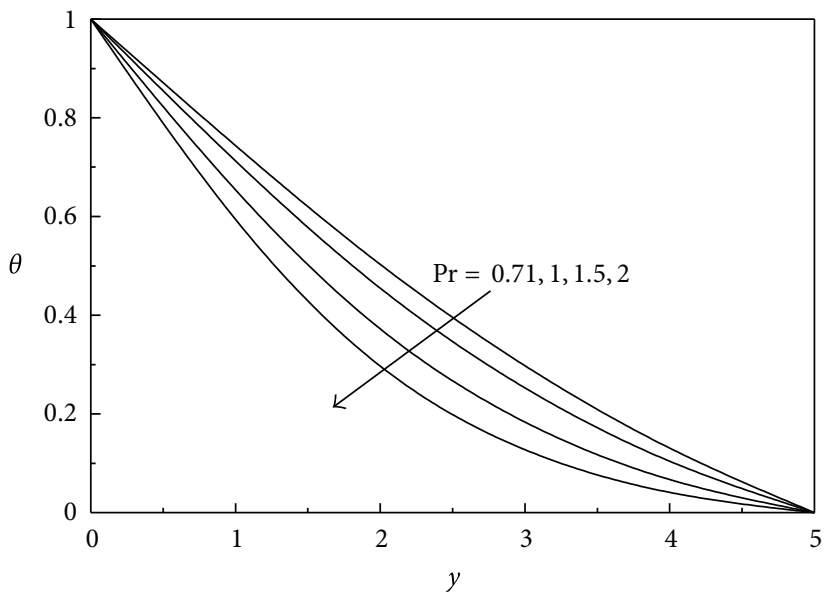

(b)

Figure 10: (a) Variation of the velocity component $f^{\prime}$ with Pr. (b) Variation of the velocity component $\theta$ with $\operatorname{Pr}$.

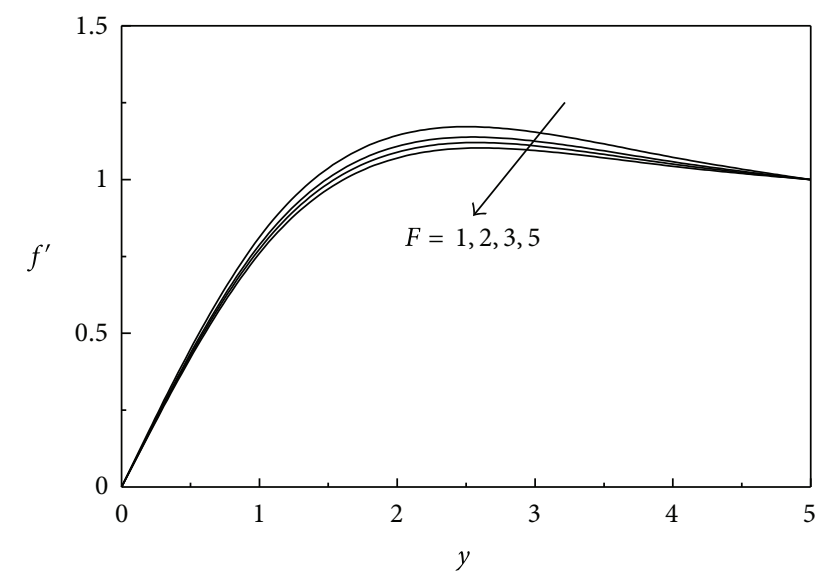

(a)

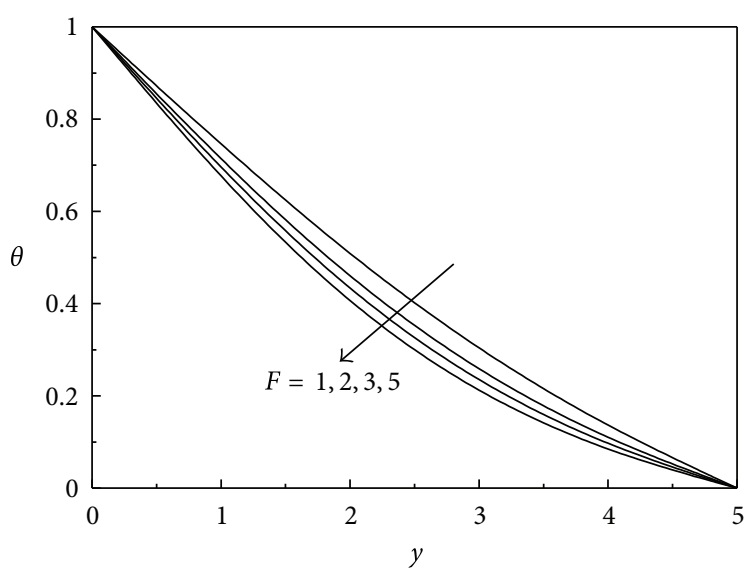

(b)

FIGURE 11: (a) Variation of the velocity component $f^{\prime}$ with $F$. (b) Variation of the velocity component $\theta$ with $F$. 


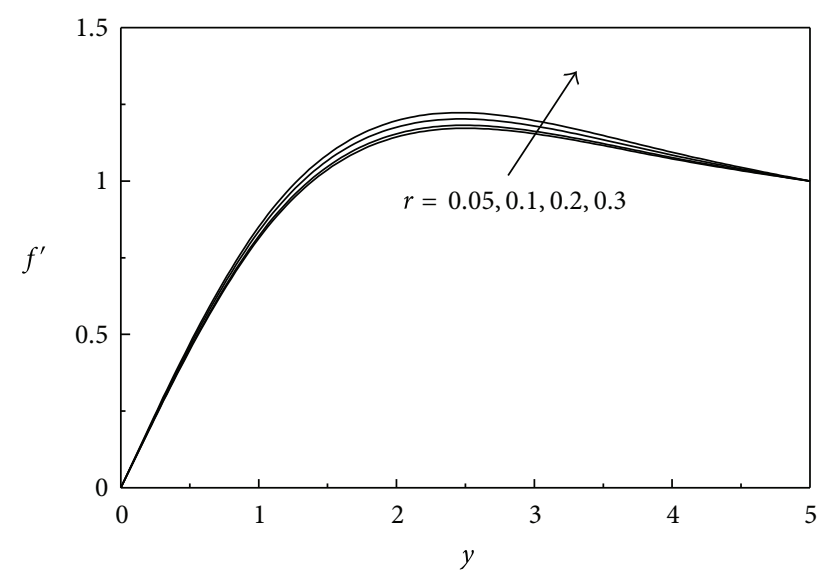

(a)

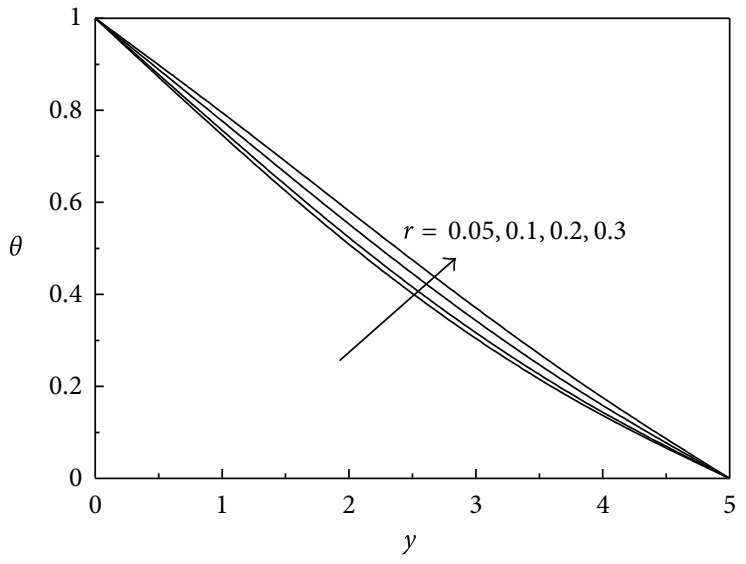

(b)

FIGURE 12: (a) Variation of the velocity component $f^{\prime}$ with $r$. (b) Variation of the velocity component $\theta$ with $r$.

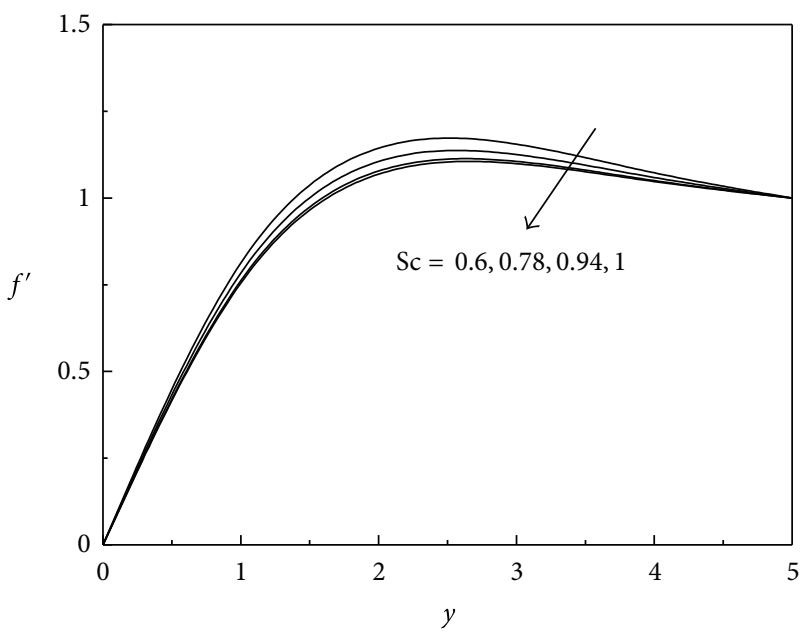

(a)

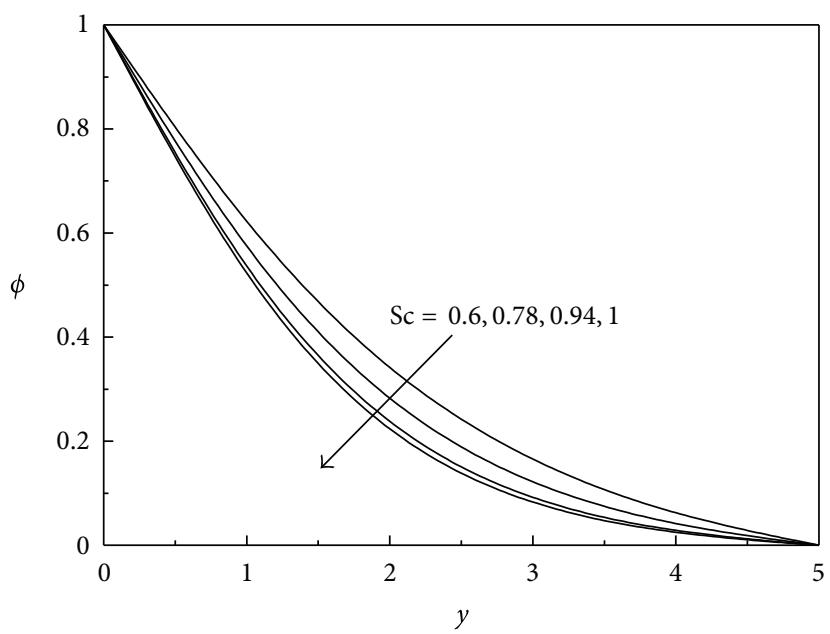

(b)

FIGURE 13: (a) Variation of the velocity component $f^{\prime}$ with Sc. (b) Variation of the velocity component $\phi$ with Sc.

momentum boundary layer thickness decreases and, therefore, an increase in the values of $f^{\prime \prime}(0)$ occurs. The results indicate that a distinct fall in the skin-friction coefficient in the $x$-direction $\left(f^{\prime \prime}(0)\right.$ and $\left.g^{\prime \prime}(0)\right)$, the surface heat transfer rate $-\theta^{\prime}(0)$, and mass transfer rate $-\phi^{\prime}(0)$, while gradient of angular velocity $h^{\prime}(0)$ increases accompanies a rise in the magnetic field parameter $M$. Increases in the values of $\mathrm{Da}$ have the effect of increasing the skin-friction function $f^{\prime \prime}(0)$, heat transfer rate $-\theta^{\prime}(0)$ and mass transfer rate $-\phi^{\prime}(0)$ while the gradient of angular velocity $h^{\prime}(0)$ and the skin-friction function $g^{\prime \prime}(0)$ slightly decreases as Da increases. Further, the influence of the porous medium inertia coefficient $\gamma$ on the wall shear stresses, gradient of angular velocity, surface heat transfer, and surface mass transfer rate is the same as that of the inverse Darcy number $\mathrm{Da}^{-1}$ since it also represents resistance to the flow. Namely, as $\gamma$ increases, $f^{\prime \prime}(0), \theta^{\prime}(0), \phi^{\prime}(0)$ decrease while $g^{\prime \prime}(0),-h^{\prime}(0)$ slightly increases, respectively.

From Table 3 for given values of Gr, Gc, $M, \mathrm{Da}, \gamma$, an increase in the values of microrotation parameter $N 1$ leads to reduction in the skin-friction function $g^{\prime \prime}(0), \theta^{\prime}(0)$ and $\phi^{\prime}(0)$ while the skin-friction function $f^{\prime \prime}(0)$ and gradient of angular velocity $h^{\prime}(0)$, increase as N1 increases. The skin friction $f^{\prime \prime}(0)$ increases and the gradient of angular velocity $h^{\prime}(0)$ is decreased as the microrotation parameter $G$ increases, while the skin-friction coefficient in the $x$-directions $g^{\prime \prime}(0)$ heat transfer rate $-\theta^{\prime}(0)$, and mass transfer rate $-\phi^{\prime}(0)$ are insensible to change in $G$. Increasing the values of heat generation parameter $Q$ results in an increase in the values 
of $f^{\prime \prime}(0)$ and the heat transfer rate $-\theta^{\prime}(0)$ decreases. It is observed that the magnitude of the wall temperature gradient increases as Prandtl number Pr or radiation parameter $F$ increases. The magnitude of the wall concentration increases with an increase in the Schmidt number Sc. Furthermore, the negative values of the wall temperature and concentration gradients, for all values of the dimensionless parameters, are indicative of the physical fact that the heat flows from the sheet surface to the ambient fluid.

\section{Conclusions}

The problem of steady, laminar, free convection boundary layer flow of micropolar fluid from a vertical stretching surface embedded in a non-Darcian porous medium in the presence of thermal radiation, mass transfer, uniform magnetic field, heat generation, and free stream velocity was investigated. A similarity transformation was employed to change the governing partial differential equations into ordinary one. These equations were solved numerically by the fourth order Rung-Kutta along with the shooting technique. A wide selection of numerical results have been presented giving the evolution of the velocity, microrotation, temperature, and concentration profiles as well as the skinfriction coefficient, heat transfer rate, and mass transfer rate. It was found that the skin-friction coefficient, heat transfer rate and mass transfer rate are decreased and the gradient of angular velocity increases as the inverse Darcy number, porous medium inertia coefficient, or magnetic field parameter is increased. Increases in the heat generation/absorption coefficient caused increases in the skin-friction coefficient and decrease the heat transfer rate. It was noticed that the increase in radiation parameter or Prandtl number caused decrease in the skin-friction coefficient and increase in heat transfer rate. In addition it was found that the increase in Schmidt number caused decrease in skin-friction coefficient and increase in mass transfer rate.

\section{Nomenclature}

\author{
$C$ : Transport property related to the inertia effect \\ $C^{\prime}$ : Fluid concentration \\ $c_{p}$ : Specific heat at constant pressure \\ Da: Darcy number \\ $f$ : Dimensionless stream function \\ $F$ : Radiation parameter \\ $G$ : Microrotation constant \\ Gc: Solutal Grashof number \\ Gr: Grashof number \\ $h$ : Dimensionless microrotation \\ $J$ : Length of microstructure \\ $k_{1}$ : Coupling constant \\ $k_{e}$ : Thermalconductivity \\ $K$ : Permeability of porous medium \\ $m$ : Boundary parameter \\ $M$ : Magnetic field parameter \\ N1: Vortes viscosity parameter \\ Pr: Prandtl number
}

$q_{r}: \quad$ Radiative heat flux

$Q_{0}$ : Heat generation constant

Q: Heat generation parameter

$r$ : Parameter of relative difference between the temperature of the sheet and temperature far away from the sheet

$T$ : $\quad$ Fluidtemperature

$T_{w}$ : Surface temperature

$T_{\infty}$ : Ambient temperature

Sc: Schmidt number

$u, v$ : Velocity components in the $x$-and $y$-directions, respectively

Uw: Velocity of the stretching sheet

$x, y$ : Cartesian coordinates along the sheet andnormal to it, respectively.

\section{Greek Letters}

$\alpha$ : Thermaldiffusivity

$\beta$ : Thermal expansion coefficient

$\gamma$ : Spin gradient viscosity

$\eta$ : Similarity variable

$\theta$ : Dimensionless temperature

$\phi$ : Dimensioless Concentration

$\kappa$ : Vortexviscosity

$\nu$ : Kinematic viscosity

$\mu$ : Dynamic viscosity

$\rho$ : Fluid density

$\sigma^{*}$ : Stefan-Boltzmann constant

$\psi$ : Stream function.

\section{Subscripts}

$w$ Condition at the solid surface $\infty$ : Ambient condition.

\section{Superscript}

( )_: Differentiatiation with respect to $\eta$.

\section{Acknowledgments}

The authors are very much thankful to the editor and the reviewers for their encouraging and constructive suggestions to improve the presentation of this paper.

\section{References}

[1] A. C. Eringen, “Theory of anisotropic micropolar fluids," International Journal of Engineering Science, vol. 18, no. 1, pp. 5-17, 1980.

[2] A. C. Eringen, “Theory of thermomicrofluids," Journal of Mathematical Analysis and Applications, vol. 38, no. 2, pp. 480-496, 1972.

[3] G. Lukaszewicz, Micropolar Fluids-Theory and Applications, Birkhäauser, Boston, Mass, USA, 1999. 
[4] T. Ariman, M. A. Turk, and N. D. Sylvester, "Microcontinuum fluid mechanics-a review," International Journal of Engineering Science, vol. 11, no. 8, pp. 905-930, 1973.

[5] G. Ahmadi, "Self-similar solution of imcompressible micropolar boundary layer flow over a semi-infinite plate," International Journal of Engineering Science, vol. 14, no. 7, pp. 639-646, 1976.

[6] S. K. Jena and M. N. Mathur, "Similarity solutions for laminar free convection flow of a thermomicropolar fluid past a nonisothermal vertical flat plate," International Journal of Engineering Science, vol. 19, no. 11, pp. 1431-1439, 1981.

[7] B. C. Sakiadis, "Boundary-layer on continuous solid surface," AIChE Journal, vol. 7, no. 1, pp. 26-28, 1961.

[8] B. C. Sakiadis, "Boundary-layer behavior on continuous moving solid surface," AIChE Journal, vol. 7, no. 2, pp. 221-225, 1961.

[9] K. R. RajaGopal, T. Y. Na, and A. S. Gupta, "A non-similar boundary layer on a stretching sheet in a non-Newtonian fluid with uniform free stream," Journal of Mathematical and Physical Sciences, vol. 21, no. 2, pp. 189-200, 1987.

[10] F. M. Hady, "On the solution of heat transfer to micropolar fluid from a non-isothermal stretching sheet with injection," International Journal of Numerical Methods for Heat and Fluid Flow, vol. 6, no. 6, pp. 99-104, 1996.

[11] T. Y. Na and I. Pop, "Boundary-layer flow of a micropolar fluid due to a stretching wall," Archive of Applied Mechanics, vol. 67, no. 4, pp. 229-236, 1997.

[12] I. Hassanien, R. S. R. Gorla, and A. A. Abdulah, "Numerical solution for heat transfer in micropolar fluids over a stretching sheet," Applied Mechanics For Engineers, vol. 3, pp. 3-12, 1998.

[13] A. Desseaux and N. A. Kelson, "Flow of micropolar fluid bounded by stretching sheet," ANZIAM Journal, vol. 42, pp. 532-560, 2000.

[14] E. M. Abo-Eldahab and A. F. Ghonaim, "Convective heat transfer in an electrically conducting micropolar fluid at a stretching surface with uniform free stream," Applied Mathematics and Computation, vol. 137, no. 2-3, pp. 323-336, 2003.

[15] K. B. Pavlov, "Magnetohydrodynamic flow of an incompressible viscous fluid caused by deformation of a surface," Magnitnaya Gidrodinamika, vol. 4, pp. 146-147, 1974.

[16] A. Chakrabarti and A. S. Gupta, "Hydromagnetic flow and heat transfer over a stretching sheet," Quarterly of Applied Mathematics, vol. 37, no. 1, pp. 73-78, 1979.

[17] E. M. Abo-Eldahab and M. A. El Aziz, "Flow and heat transfer in a micropolar fluid past a stretching surface embedded in a nonDarcian porous medium with uniform free stream," Applied Mathematics and Computation, vol. 162, no. 2, pp. 881-899, 2005.

[18] A. Bejan and K. R. Khair, "Heat and mass transfer by natural convection in a porous medium," International Journal of Heat and Mass Transfer, vol. 28, no. 5, pp. 909-918, 1985.

[19] C. T. Hsu and P. Cheng, "The Brinkman model for natural convection about a semi-infinite vertical flat plate in a porous medium," International Journal of Heat and Mass Transfer, vol. 28, no. 3, pp. 683-697, 1985.

[20] C. L. Tien and K. Vafai, "Convective and radiative heat transfer in porous media," Advances in Applied Mechanics, vol. 27, pp. 225-281, 1989.

[21] D. Pal and S. Chatterjee, "Heat and mass transfer in MHD non-Darcian flow of a micropolar fluid over a stretching sheet embedded in a porous media with non-uniform heat source and thermal radiation," Communications in Nonlinear Science and Numerical Simulation, vol. 15, no. 7, pp. 1843-1857, 2010.
[22] M. A. A. Mahmoud, "Heat generation/absorption and viscous dissipation effects on MHD flow of a micropolar fluid over a moving permeable surface embedded in a Non-darcian porous medium," Journal of the Korean Physical Society, vol. 54, no. 4, pp. 1526-1531, 2009.

[23] A. Nakayama and H. Koyama, "Similarity solutions for buoyancy-induced flows over a non-isothermal curved surface in a thermally stratified porous medium," Applied Scientific Research, vol. 46, no. 4, pp. 309-322, 1989.

[24] A. Ishak, "Thermal boundary layer flow over a stretching sheet in a micropolar fluid with radiation effect," Meccanica, vol. 45, no. 3, pp. 367-373, 2010.

[25] M. E. M. Khedr, A. J. Chamkha, and M. Bayomi, "MHD flow of a micropolar fluid past a stretched permeable surface with heat generation or absorption," Nonlinear Analysis: Modelling and Control, vol. 14, no. 1, pp. 27-40, 2009.

[26] E. M. Abo-Eldahab and A. F. Ghonaim, "Radiation effect on heat transfer of a micropolar fluid through a porous medium," Applied Mathematics and Computation, vol. 169, no. 1, pp. 500-510, 2005.

[27] M. Gnaneswara Reddy, "Heat generation and thermal radiation effects over a stretching sheet in a micropolar fluid," ISRN Thermodynamics, vol. 2012, Article ID 795814, 6 pages, 2012.

[28] P. O. Olanrewaju, G. T. Okedayo, and J. A. Gbadeyan, "Effects of thermal radiation on magnetohydrodynamic flow of a micropolar fluid towards a stagnation point on a vertical plate," International Journal of Applied Science and Technology, vol. 1, no. 6, pp. 1-6, 2011.

[29] A. J. Willson, "Boundary layer in micropolar liquids," Mathematical Proceeding of the Cambridge Philosophical Society, vol. 67, no. 2, pp. 469-476, 1970.

[30] D. A. Nield and A. Bejan, Convection in Porous Media, Springer, New York, NY, USA, 1999.

[31] M. Q. Brewster, Thermal Radiative Transfer and Properties, John Wiley \& Sons, New York, NY, USA, 1992.

[32] L. Rosenhead, Laminar Boundary, Oxford University Press, Oxford, UK, 1963.

[33] B. Carnahan, H. A. Luther, and O. W. James, Applied Numerical Methods, John Wiley \& Sons, New York, NY, USA, 1969. 

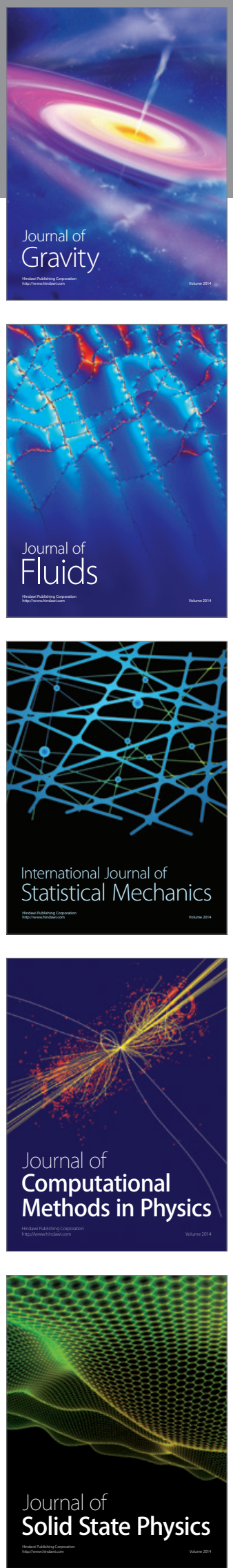

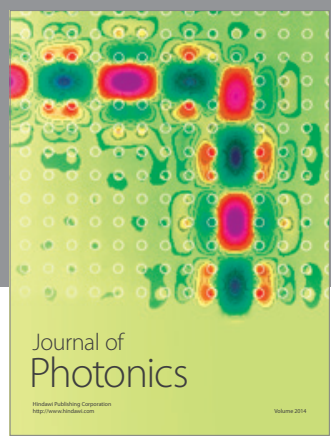

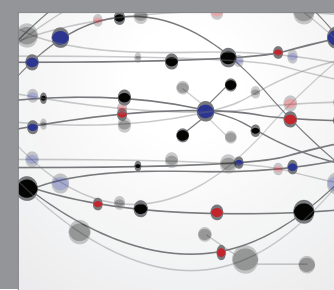

The Scientific World Journal

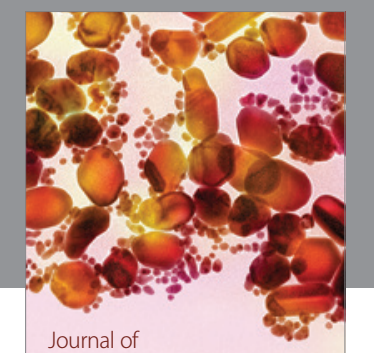

Soft Matter
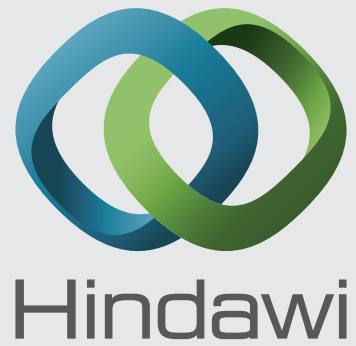

Submit your manuscripts at

http://www.hindawi.com
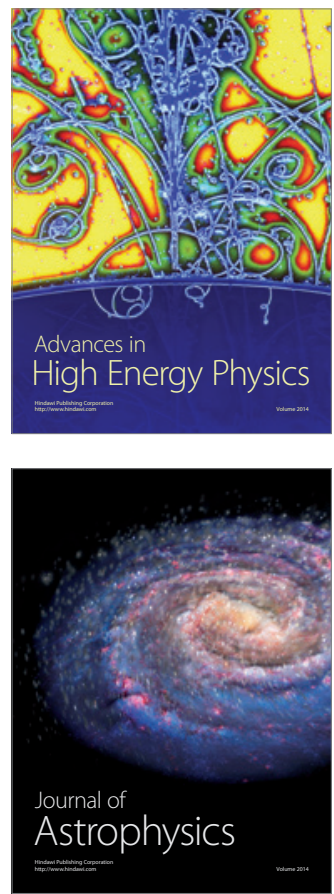
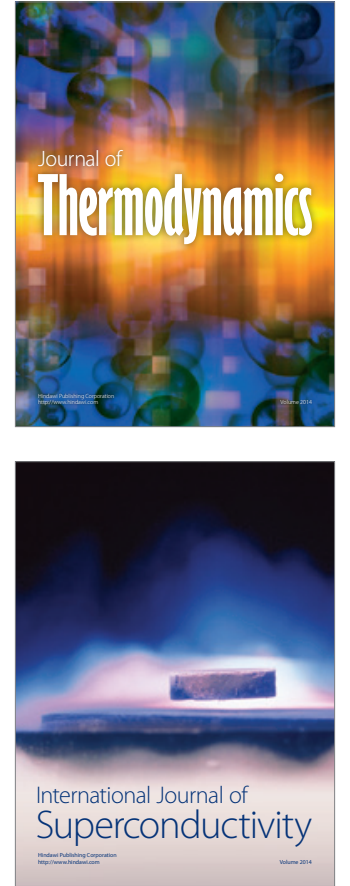
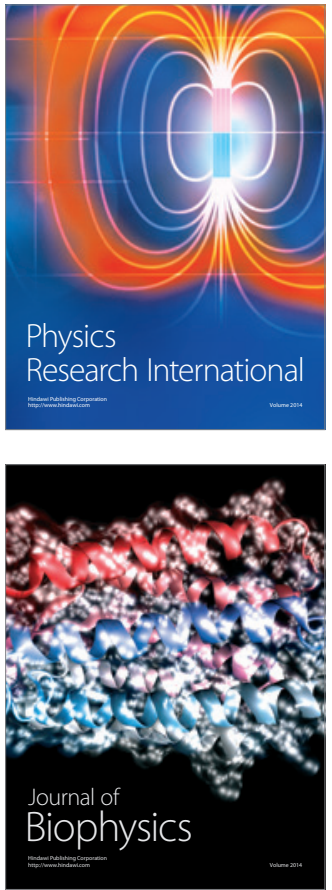
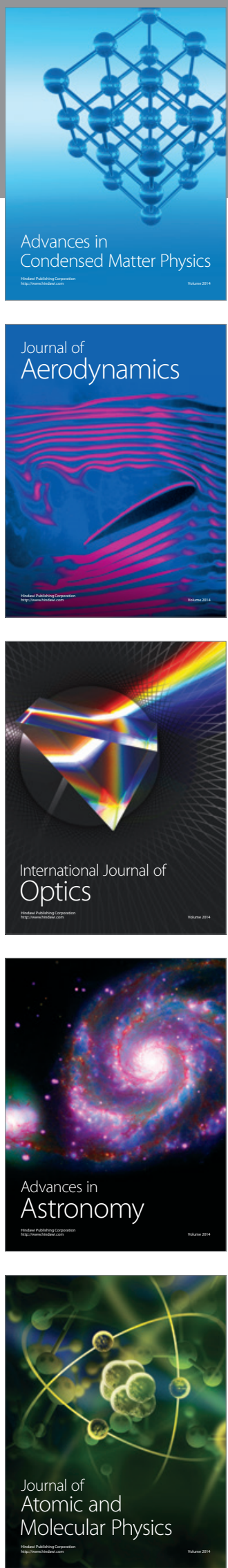